\title{
DISEÑO POR DESEMPEÑO DE ESTRUCTURAS DÚCTILES DE CONCRETO REFORZADO UBICADAS EN LA ZONA DEL LAGO DEL DISTRITO FEDERAL: EJEMPLO DE APLICACIÓN
}

\author{
Alberto Sánchez Badillo ${ }^{(1)}$ y Amador Terán Gilmore ${ }^{(2)}$
}

\begin{abstract}
RESUMEN
El desempeño no satisfactorio de algunas estructuras diseñadas conforme a reglamentos actuales de diseño sísmico ha preocupado a la comunidad internacional de ingeniería estructural. Debido a las grandes pérdidas económicas derivadas de eventos sísmicos recientes se han formulado metodologías de diseño basadas en el control explícito de la respuesta dinámica de las estructuras. Este artículo introduce, dentro del marco del denominado diseño por desempeño, una metodología numérica de diseño sísmico que integra la fase de prediseño y de diseño local, y que considera los siguientes aspectos: 1) el desempeño estructural y no estructural de la edificación, 2) un enfoque dual que considera los estados límite de servicio y seguridad, y 3) en cuanto el estado límite de seguridad, la fatiga de bajo número de ciclos a través de espectros de resistencia de ductilidad acumulada constante, y el efecto del deterioro del ciclo histerético en la definición de los espectros de resistencia y desplazamiento.
\end{abstract}

\begin{abstract}
The unsatisfactory performance of some structures designed according to current seismic design codes has raised some concern in the international community of structural engineers. Due to the large economical losses derived from recent seismic events, design methodologies that are based in the explicit control of the dynamic response of structures have been formulated. This paper introduces, within the framework of performance-based design, a seismic design numerical methodology that integrates the predesign and local design steps, and that considers the following aspects: 1) the structural and non-structural performance of the building, 2) a dual approach that considers the service and life safety limit states, and 3) regarding the life safety limit state, low cycle fatigue through the use of cumulative ductility spectra, and the effect of degradation of the hysteretic cycle in the definition of strength and displacement spectra.
\end{abstract}

\section{INTRODUCCIÓN}

El desempeño sísmico insatisfactorio de algunas estructuras diseñadas conforme a los reglamentos actuales de diseño sísmico ha preocupado al medio de la ingeniería estructural. Esto ha cobrado particular

Artículo recibido el 10 de marzo de 2006 y aprobado para su publicación el 30 de noviembre de 2007. Se aceptarán comentarios y/o discusiones hasta cinco meses después de su publicación.

(1) Alonso y Asociados, Carretera México-Toluca 1725, Despacho C-5, Col. Lomas de Palo Alto, México 05110, D.F., alberto@alonsoasociados.com.mx

(2) Departamento de Materiales, Universidad Autónoma Metropolitana, Av. San Pablo 180, Col. Reynosa Tamaulipas, México 02200, D.F., tga@correo.azc.uam.mx 
importancia a partir de las grandes pérdidas materiales y económicas que han resultado de eventos sísmicos severos, como los de México 1985, Loma Prieta 1989, Northridge 1994 y Kobe 1995. Dado el nivel inesperadamente alto de las pérdidas, se han formulado enfoques integrales de diseño sísmico, tal como el de diseño por desempeño, que enfatizan la necesidad de un control explícito de la respuesta dinámica de la estructuras.

Paulatinamente, diferentes reglamentos a nivel mundial han ido incorporando conceptos de Diseño por Desempeño. México ha fomentado la transparencia en el diseño sísmico a través del Apéndice A de las Normas Técnicas Complementarias para Diseño por Sismo (NTCDS) del Reglamento de Construcciones del D.F. (Gobierno del Distrito Federal 2004a). Independientemente del avance conceptual y numérico que el Apéndice A representa, es necesario reconocer que falta mucho por hacer para racionalizar el diseño sísmico de las estructuras dúctiles desplantadas en la Zona del Lago del D.F., y que esto representa un gran reto para los investigadores y profesionales mexicanos del diseño sísmico.

Este artículo plantea, dentro del marco propuesto por el Comité Visión 2000 (SEAOC 1995) para un diseño sísmico por desempeño, una metodología numérica de diseño para marcos dúctiles de concreto reforzado (Sánchez 2005). La metodología propuesta considera explícitamente los estados límite de servicio y seguridad y se enfoca al diseño de estructuras dúctiles ubicadas en la Zona del Lago del Distrito Federal. Dado las particularidades de los movimientos generados en dicha zona, la metodología propuesta hace consideración explícita de la fatiga de bajo número de ciclos, y del efecto de la degradación del ciclo histerético. En cuanto a la fatiga, se considera el uso de espectros de ductilidad acumulada constante para plantear el control de las demandas máxima y acumulada de deformación plástica. La degradación del ciclo histerético se contempla a partir de modificar, mediante una serie de reglas sencillas, los espectros de resistencia y desplazamiento derivados de sistemas de un grado de libertad con comportamiento elastoplástico perfecto. Las bases en las que se apoya la metodología propuesta se discuten en mayor detalle en el artículo que acompaña al presente dentro de esta revista (Terán y Espinoza 2008).

Se ilustra además la aplicación de la metodología propuesta para el diseño de un edificio de doce pisos. Vale la pena hacer notar que la metodología está enfocada al diseño de marcos dúctiles de concreto reforzado, y que para ampliar el alcance de la misma habría que considerar su calibración para otros materiales y sistemas estructurales, y refinar algunas de las herramientas que su aplicación involucra. Se entiende como marco dúctil de concreto reforzado aquel que cumple con los requerimientos de regularidad estipulados en las NTCDS para un $Q$ de 4 , y los requisitos de detallado y de resistencia relativa entre vigas, columnas y conexiones, estipulados por las Normas Técnicas Complementarias para Diseño y Construcción de Estructuras de Concreto (NTCDCEC) del Reglamento de Construcciones del D.F. (Gobierno del Distrito Federal 2004b).

\section{DISEÑO POR DESEMPEÑO}

En los últimos años, varios investigadores y profesionistas han empezado a hablar del diseño basado en el desempeño de las estructuras, como una manera más racional para plantear el problema del diseño sísmico. El Comité Visión 2000 (SEAOC 1995), ha propuesto un proceso global de diseño que consta de tres fases:

- Fase Conceptual. Se enfoca a la concepción de una solución estructural y no estructural al problema de diseño planteado. Su primer paso consiste en el planteamiento de los objetivos de diseño, que deben reflejar de manera clara las expectativas que surgen de la construcción de la obra. Luego se establece, acorde a la sismicidad del sitio, si lo obra es técnica y económicamente viable. De ser viable, el ingeniero estructural debe plantear el Diseño Conceptual de acuerdo con 
los objetivos de diseño y con las características de los movimientos sísmicos esperados. Durante el Diseño Conceptual deben establecerse la configuración global de la construcción, la configuración estructural, los sistemas y materiales estructurales, el sistema de cimentación y el tipo de elementos no estructurales y su conexión a la estructura.

- Fase Numérica. Está constituida por dos etapas (Diseño Preliminar y Diseño Final) que involucran el dimensionado y detallado de los sistemas estructurales y no estructurales.

- Implantación. Garantiza la calidad del diseño mediante una revisión detallada e independiente. Además, el diseño por desempeño no puede ser exitoso sin un control de calidad adecuado durante la construcción de la estructura, y sin una supervisión adecuada y continua del mantenimiento, ocupación y función de la misma.

Pueden distinguirse tres etapas durante la Fase Numérica de las metodologías de diseño que se han propuesto recientemente para controlar de manera explícita la respuesta dinámica de las estructuras (Terán 2004):

- Prediseño global. Con la ayuda de espectros de respuesta, se determinan a nivel global las características mecánicas relevantes de la estructura de manera que su respuesta dinámica global durante la excitación sísmica de diseño no exceda los límites de respuesta establecidos en función de los criterios de desempeño.

- Diseño local preliminar. Una vez establecidas las características mecánicas a nivel global, se procede al diseño local de la estructura, donde se determinan, en función de dichas características, las dimensiones y detallado de los elementos estructurales.

- Revisión del diseño. Se ha planteado una serie de lineamientos para la revisión del diseño preliminar de la estructura a través de una serie de análisis estructurales con alto grado de refinamiento (especialmente si la estructura debe exhibir comportamiento plástico).

Es importante recordar que en ocasiones una metodología numérica de diseño debe considerar explícitamente más de un objetivo de diseño. Dentro de este contexto, cada objetivo debe considerar explícitamente la representación numérica (espectros) del nivel sísmico de diseño que le corresponde. Para cada objetivo de diseño es necesario determinar las características mecánicas requeridas para satisfacer su criterio de desempeño, y diseñar los elementos estructurales y no estructurales acorde a la condición crítica que resulte de todos los objetivos de diseño relevantes.

Dentro del contexto del diseño por desempeño, las características mecánicas deben suministrarse a una estructura de manera que, dentro de límites técnicos y económicos aceptables, sean capaces de controlar y acomodar su respuesta dinámica dentro de umbrales congruentes con el nivel de daño aceptable. En particular, el control del daño estructural y no estructural requiere del control de la distorsión máxima de entrepiso y en ocasiones, como es el caso de edificaciones construidas en la Zona del Lago del D.F., de la consideración explícita de las demandas acumuladas de deformación plástica (Terán y Espinoza 2008). En cuanto al daño en instalaciones y contenidos, puede llegar a ser necesario plantear el control de las demandas de velocidad y aceleración de entrepiso.

La metodología de diseño sísmico introducida en este artículo se enmarca dentro del planteamiento que para el diseño por desempeño ha desarrollado el Comité Visión 2000, particularmente dentro de las etapas de Prediseño Global y de Diseño Local Preliminar de la Fase Numérica. 


\section{CONSIDERACIONES DE DESEMPEÑO PARA LA CIUDAD DE MÉXICO}

Un diseño sísmico por desempeño debe considerar el control del nivel de daño estructural y no estructural en las estructuras cuando se ven sujetas a las excitaciones sísmicas de diseño de diferente intensidad. Ha sido común que las metodologías numéricas de diseño por desempeño planteen controlar el desplazamiento lateral máximo de la estructura sismorresistente (Qi y Moehle 1991, Priestley 2000). Esto se debe a que los umbrales de deformación máxima planteados por los enfoques de diseño por desplazamiento parecen lo suficientemente conservadores para garantizar un diseño sísmico adecuado de las estructuras desplantadas en suelo firme (Terán y Jirsa 2003). Sin embargo, es necesario reconocer que los movimientos de terreno generados en la Zona del Lago del D.F. presentan particularidades que implican un tratamiento ligeramente diferente al control del desplazamiento lateral. En particular, se ha observado que tanto las demandas acumuladas de comportamiento plástico como la degradación del ciclo histerético impactan de manera importante la respuesta dinámica, y por tanto el desempeño sísmico, de las estructuras dúctiles ubicadas en dicha zona. En esta sección se esbozan herramientas numéricas que hacen posible la incorporación de estos dos aspectos a una metodología de diseño por desempeño. Para una discusión más detallada, se refiere al lector al artículo que acompaña al presente dentro de esta revista (Terán y Espinoza 2008).

\section{Modelos de fatiga de bajo número de ciclos (índices de daño)}

En este artículo se utilizan 2 modelos de daño que contemplan explícitamente el efecto de las demandas acumuladas de comportamiento plástico. El primer modelo, planteado por Terán y Jirsa (2003), se usa dentro de la metodología propuesta a través del concepto de espectro de ductilidad acumulada constante (Terán y Espinoza 2008). El segundo modelo, planteado por Park y Ang (1985), se utiliza para evaluar a nivel local el nivel de daño estructural del edificio de doce pisos diseñado de acuerdo a la metodología propuesta.

\section{Efecto de la degradación del ciclo histerético}

La metodología propuesta en este artículo se centra en el diseño de estructuras de concreto reforzado, las cuales exhiben degradación importante de sus características mecánicas en función de sus demandas de deformación plástica. En el caso de estructuras dúctiles de concreto reforzado, dicha degradación puede caracterizarse a través de la pérdida de rigidez lateral que se observa conforme se incrementa su nivel de deformación plástica (tanto máxima como acumulada). Una forma de tomar en cuenta este tipo de degradación durante el diseño sísmico consiste en modificar, mediante el uso de factores correctivos, las demandas sísmicas (por lo general de resistencia y desplazamiento) obtenidas a partir de espectros establecidos para comportamiento elasto-plástico perfecto. Los factores de corrección utilizados aquí para el diseño del edificio de doce pisos son los propuestos por Terán y Espinoza (2008).

\section{METODOLOGÍA PROPUESTA}

Los reglamentos actuales de diseño sísmico han incorporado gradualmente algunos conceptos de diseño sísmico por desempeño. Aunque el Reglamento de Construcciones para el Distrito Federal ha sido sujeto a una etapa de modernización, es necesario actualizar algunos de sus requerimientos de diseño sísmico para poder plantear cabalmente a través de él un diseño basado en el control de la respuesta sísmica. 


\section{Planteamiento general}

La metodología discutida en este artículo, aplicable al diseño de estructuras de ocupación estándar, considera los estados límite de servicio y seguridad de vida; y se enmarca dentro de la Fase Numérica del Diseño por Desempeño. En lo particular, dicha metodología contempla las fases de Prediseño Global y Diseño Local Preliminar. Vale la pena mencionar que el planteamiento no contempla explícitamente la Fase Conceptual del Diseño por Desempeño. En otras palabras, no se discutirán las consideraciones que deben hacerse para establecer los objetivos de diseño ni la determinación de las excitaciones sísmicas de diseño. Se supondrá que una vez resuelta la Fase Conceptual del diseño, el ingeniero ha decidido estructurar la edificación a través de marcos dúctiles de concreto reforzado, que el diseño requiere revisar explícitamente los estados límite de servicio y seguridad, y que es de relevancia revisar tanto el desempeño estructural como el no estructural.

\section{Diseño preliminar}

Un buen diseño preliminar de las estructuras sismorresistentes no solo conduce a una estructura con un desempeño aceptable, sino a una solución eficiente. Un diseño preliminar deficiente puede verse fácilmente reflejado en un diseño final limitado y muy lejano a una solución óptima. La Figura 1 resume esquemáticamente los pasos de la metodología que aquí se propone para llevar a cabo el Prediseño Global.

El primer paso consiste en establecer, a través de los objetivos de diseño, una definición cualitativa del comportamiento deseado de la estructura. Esto se hace a través de la consideración explícita de los niveles de daño aceptable según los estados límite bajo consideración. El segundo paso consiste en la cuantificación del comportamiento deseado a través de establecer umbrales de respuesta en la estructura con la ayuda de índices de daño. Durante el tercer paso, la metodología establece el valor de las características mecánicas de la estructura a nivel global a través de definir, con la ayuda de espectros de resistencia y desplazamiento, el valor de tres parámetros globales de diseño: el cortante basal, que cuantifica la resistencia lateral de diseño; el periodo fundamental de vibración; que cuantifica los requerimientos de diseño de rigidez lateral; y la ductilidad última, estimada a partir del desplazamiento lateral de azotea y que caracteriza la capacidad de deformación última de la estructura. Conforme a lo discutido con anterioridad, el valor de estas características mecánicas debe ser tal que la estructura sea capaz de controlar y acomodar, dentro de límites técnicos y económicos aceptables, su respuesta dinámica dentro de umbrales congruentes con el nivel de daño aceptable. Una vez establecidos los valores de estos tres parámetros, durante el cuarto paso se procede al diseño local de resistencia, rigidez y capacidad de deformación de los elementos estructurales. Mientras que los tres primeros pasos de la metodología se enmarcan dentro de la etapa de Prediseño Global, el cuarto paso corresponde a la etapa de Diseño Local Preliminar.

El proceso de diseño sísmico inicia a través de la Fase Conceptual. Se ha planteado que para este artículo, el diseño conceptual ha arrojado, de alguna manera, que la edificación se estructurará a través de marcos dúctiles de concreto reforzado. El valor del primer parámetro de diseño, que caracteriza la capacidad global de deformación última de la estructura, puede establecerse en función del sistema estructural y detallado propuesto. Para un marco dúctil de concreto reforzado, la evidencia experimental sugiere que la ductilidad última bajo deformación monótonamente creciente, $\mu_{u}$, oscila entre 4 y 6 . En este estudio se considera un valor intermedio $\mu_{u}=5$. 


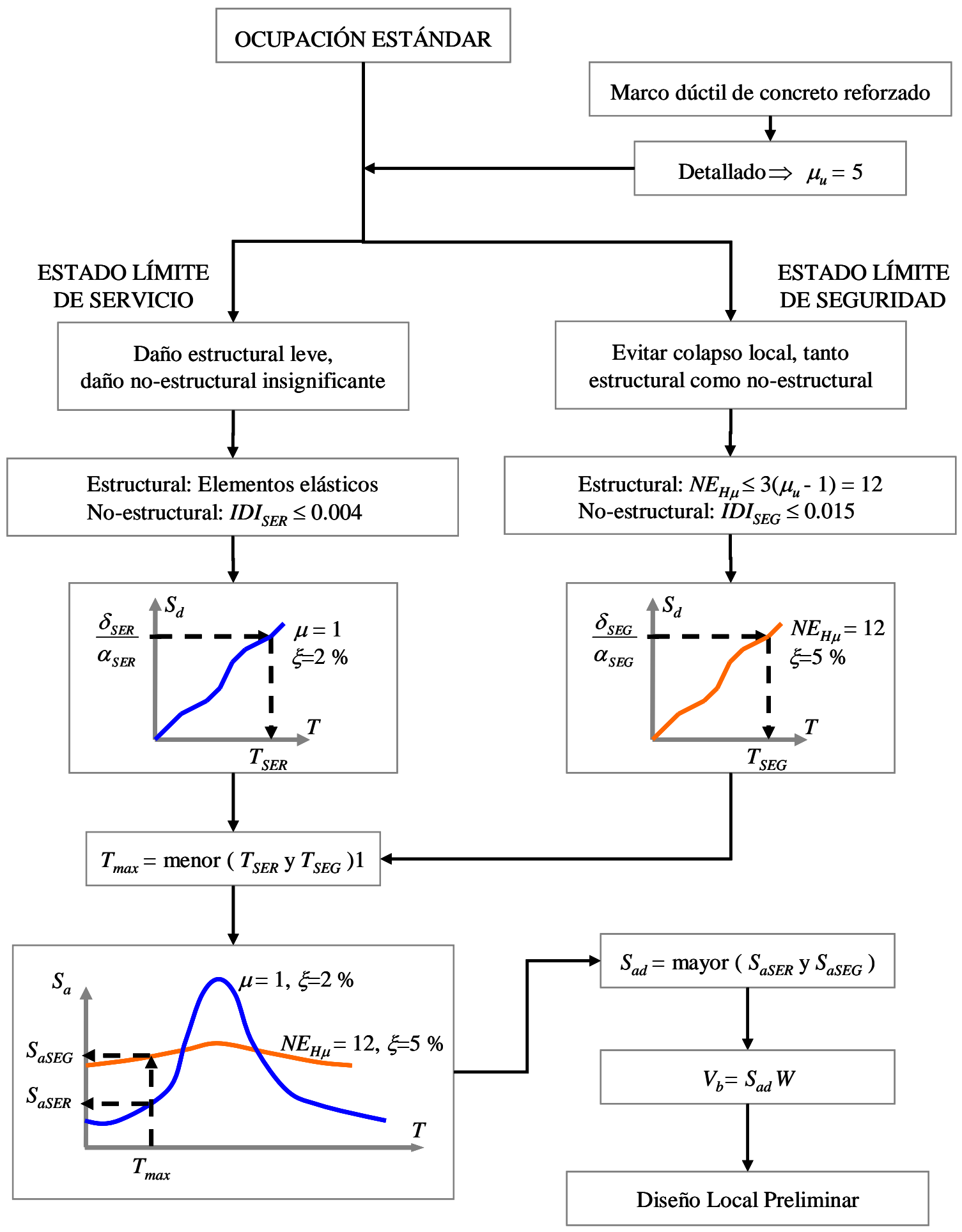

Figura 1. Prediseño Global 
En cuanto a las definiciones cualitativas asociadas a los estados límite bajo consideración, el estado límite de servicio se considera satisfecho si: 1) Los elementos estructurales no exhiben disminución de su capacidad resistente y de deformación última (aunque con posible pérdida de rigidez debido a su agrietamiento), y 2) Los elementos no estructurales permanecen prácticamente sin daño, de manera que la estructura permanezca operable después de la excitación sísmica. En cuanto a la cuantificación del estado límite de servicio, se considera que los elementos estructurales satisfacen sus criterios de desempeño mientras permanezcan elásticos, y que el daño en los elementos no estructurales se controla dentro de los límites bajo consideración si a su vez se controla la distorsión máxima de entrepiso $\left(I D I_{S E R}\right)$ dentro del umbral de 0.004. Note que el Apéndice A de las NTCDS plantea un umbral de distorsión de 0.004 para la revisión del estado límite de servicio para estructuras en que no haya elementos no-estructurales incapaces de soportar deformaciones apreciables, o éstos estén separados de la estructura principal de manera que no sufran daños por sus deformaciones.

El estado límite de seguridad de vida se avoca a garantizar la seguridad física de los ocupantes de la estructura a través de prevenir colapsos locales de elementos estructurales y no estructurales. Acorde a la metodología propuesta, esto implica controlar: 1) El daño estructural a través de establecer un umbral para la demanda de energía normalizada en la estructura conforme al criterio de Terán y Jirsa $\left[N E_{H \mu}=3\left(\mu_{u}-1\right)=12\right.$ ], y 2) El daño no estructural a través de establecer un umbral de 0.015 para la distorsión máxima en el edificio $\left(I D I_{S E G}\right)$. Vale la pena mencionar que los umbrales de $I D I_{S E R}$ y $I D I_{S E G}$ deben establecerse en función del tipo de elementos no estructurales que se encuentran en la edificación y la manera en que éstos se conectan a la estructura. Reyes (2000) presenta umbrales de distorsión para diferentes condiciones de desempeño y tipo y conexión de elementos no estructurales.

El valor del segundo parámetro de diseño, que es el periodo fundamental de vibración del edificio, se establece, conforme a lo ilustrado en la Figura 1, a partir de umbrales de desplazamiento de azotea para los estados límites de servicio y seguridad ( $\delta_{S E R}$ y $\delta_{S E G}$, respectivamente) y espectros de desplazamiento para dichos estados límite. El umbral de desplazamiento de azotea puede establecerse conforme a lo siguiente (Qi y Moehle 1991, Terán 2004):

$\delta_{\max }=\frac{I D I_{\max } H}{C O D}$

donde $I D I_{\max }$ es la distorsión máxima permisible, $H$ la altura total del edificio a partir del nivel de suelo, y $C O D$ un factor que contempla que la distorsión no es constante en todos los entrepisos del edificio. Antes de utilizar el valor de $\delta_{\max }$ para estimar el valor del periodo fundamental de vibración de la estructura, es necesario considerar que la edificación es un sistema de varios grados de libertad, y que un espectro de desplazamientos resume resultados derivados de sistemas de un grado de libertad. Conforme a lo mostrado en la Figura 1, el valor de $\delta_{\max }$ debe modificarse para contemplar el efecto de varios grados de libertad a través del parámetro $\alpha$ (para mayor discusión, veáse Terán 2004). El espectro de desplazamientos para el estado límite de servicio contempla comportamiento elástico y un porcentaje de amortiguamiento crítico ( $\xi$ ) de $2 \%$. Para el estado límite de seguridad, se plantea el uso de un espectro de desplazamientos para ductilidad acumulada constante correspondiente a $N E_{H \mu}=3\left(\mu_{u}-1\right)=12$ y $\xi$ de $5 \%$. En cuanto al concepto de espectro para ductilidad acumulada constante, se refiere al lector a las discusiones detalladas que alrededor del mismo han formulado Terán y Jirsa (2004) y Terán y Espinoza (2008). En cuanto a los valores de porcentaje de amortiguamiento crítico asignados a los espectros, es importante que dicho 
porcentaje sea congruente con el nivel de esfuerzos esperado en los elementos estructurales. Chopra (2001) sugiere que el amortiguamiento tiende a crecer de una manera importante conforme el nivel de esfuerzos en un elemento estructural se incrementa. En particular, si el nivel de esfuerzos es del orden o menor que el $50 \%$ del esfuerzo de fluencia, el coeficiente equivalente de amortiguamiento puede ser sustancialmente menor al 5\%; mientras que si el nivel de esfuerzos corresponde a la fluencia del elemento estructural, el amortiguamiento suele ser mayor que $5 \%$. Se considera que los porcentajes de $2 \%$ y $5 \%$ asociados a los estados límite de servicio y seguridad, respectivamente, son valores razonablemente conservadores asociados al estado de daño estructural asociado a cada uno de dichos estados límite.

La Figura 1 indica que el valor de periodo fundamental de vibración $\left(T_{\max }\right)$ para el que debe diseñarse la edificación corresponde al menor de los valores dados por $T_{S E R}$ y $T_{S E G}$, que a su vez corresponden a los valores de $T$ que satisfacen los requerimientos de diseño impuestos por los estados límite de servicio y seguridad, respectivamente. Vale la pena mencionar que el menor valor de $T_{S E R}$ y $T_{S E G}$ dará lugar a la condición crítica de diseño solo cuando la rigidez lateral elástica asociada a ambos estados límite sea similar. En el planteamiento que se hace en este artículo, se considera que $I D I_{S E R}=0.004$. Para contextualizar este valor de distorsión, la evidencia experimental y analítica reportada por Reyes (2000) sugiere que un marco dúctil de concreto reforzado exhibe fluencia incipiente para distorsiones cercanas a 0.005 , de tal manera que $I D I_{S E R}$ de 0.004 implica agrietamiento importante de los elementos estructurales del marco dúctil. Dado que para el estado límite de seguridad se espera que los elementos estructurales del marco estén totalmente agrietados, puede concluirse que el valor de $I D I_{S E R}$ adoptado en este artículo implica que la rigidez elástica lateral de la estructura será similar para ambos estados límite bajo consideración. Bajo estas circunstancias, el dimensionado de los elementos estructurales queda definido por el menor de los valores de $T_{S E R}$ y $T_{S E G}$.

No siempre el menor de los valores de $T_{S E R}$ y $T_{S E G}$ da lugar a la condición crítica de diseño. Por ejemplo considere el caso en que el estado límite de servicio está asociado a menores niveles de distorsión, de tal manera que el estado esperado en los elementos estructurales corresponda a un nivel menor de agrietamiento. Bajo estas circunstancias la rigidez lateral elástica asociada al estado límite de servicio sería mayor que la que corresponde al estado límite de seguridad, y entonces sería posible encontrar casos en que la condición crítica para el dimensionado de los elementos estructurales surgiera del estado límite de seguridad a pesar de que $T_{S E G}$ fuera mayor que $T_{S E R}$ (Bertero y Bertero 1992).

Una vez que se establece el valor de $T_{\max }$, se procede a determinar el valor del tercer y último parámetro de diseño: el cortante basal. Como se muestra, el coeficiente sísmico de diseño $\left(S_{a d}\right)$ corresponde al mayor de los coeficientes sísmicos establecidos para los estados límite de servicio y seguridad ( $S_{a S E R}$ y $S_{a S E G}$, respectivamente). En caso de que se utilice el método estático de diseño, el cortante basal de diseño $\left(V_{b}\right)$ queda definido por $V_{b}=S_{a d} W$ (donde $W$ es el peso total reactivo de la estructura). Note que en el caso del estado límite de servicio, el coeficiente sísmico de diseño se estima a partir de evaluar para un valor de $T_{\max }$ un espectro elástico de pseudo-aceleración para $\xi$ de 0.02 ; y que el correspondiente a seguridad de vida se obtiene al evaluar, para ese mismo valor de periodo, un espectro de ductilidad acumulada constante para $N E_{H \mu}=12$ y $\xi$ de 0.05 . En caso de que la estructura amerite la realización de un análisis dinámico, entonces se procedería a seleccionar el espectro de diseño de resistencia entre los espectros asociados a los estados límite de servicio y seguridad. Un criterio para discernir cual espectro seleccionar sería considerar el espectro que arroje la mayor ordenada para el valor de $T_{\max }$. Debe mencionarse que el criterio delineado es particularmente válido si la respuesta dinámica del edificio está dominada por su modo fundamental de vibración, lo que en la Zona del Lago del D.F. solo no sucede en el caso de estructuras de gran altura. Bertero y Bertero (1992) hacen notar que en el caso en que los modos superiores de la estructura lleguen a contribuir de manera importante en el diseño de los 
elementos estructurales, es posible que el diseño en los pisos inferiores e intermedios esté regido por el espectro de resistencia de un estado límite, y el diseño de los pisos superiores por el otro estado límite.

Una vez concluido el Prediseño Global, se pasa a la etapa de Diseño Local Preliminar. Esta etapa se explicará en detalle conforme se desarrolle el ejemplo de aplicación.

\section{Espectros de diseño}

Para la obtención de los espectros de diseño se establecieron dos grupos de acelerogramas registrados en la Zona del Lago del D.F., y caracterizados por tener un periodo dominante de excitación $\left(T_{g}\right)$ de $2 \mathrm{seg}$. El grupo de acelerogramas correspondientes a la excitación sísmica de diseño para el estado límite de seguridad agrupa los 6 acelerogramas incluidos en la Tabla 1, los cuales fueron escalados para que exhibieran una velocidad máxima del terreno igual a la de movimiento del terreno registrado durante 1985 en la Secretaría de Comunicaciones y Transportes, dirección este-oeste (SCTEO 1985). En cuanto al estado límite de servicio, se agruparon los 12 acelerogramas contenidos en la Tabla 2. Los acelerogramas en este segundo grupo se escalaron de tal manera que exhibieran una velocidad máxima del terreno igual a la cuarta parte de la velocidad máxima del terreno correspondiente a SCTEO 1985. Los espectros de diseño corresponden a la media más una desviación estándar $(\sigma)$ de los espectros correspondientes a cada acelerograma dentro de un grupo, y se obtuvieron a partir de comportamiento elasto-plástico perfecto, de tal manera que sus ordenadas deben ajustarse para considerar explícitamente el comportamiento degradante de la estructura dúctil de concreto reforzado.

\section{EJEMPLO DE APLICACIÓN}

La Figura 2 muestra la planta y elevación del edificio considerado en este artículo para ilustrar el uso de la metodología que aquí se propone. El edificio es regular (simétrico) en cuanto a masa, resistencia y rigidez; tiene una planta definida por 3 crujías de $7 \mathrm{~m}$ de claro en cada una de las dos direcciones principales, y cuenta con doce pisos con altura de entrepiso de $4 \mathrm{~m}$, excepto para la planta baja que exhibe una altura de $5 \mathrm{~m}$. Para simplificar el diseño, se han asignado las mismas dimensiones y armado a los elementos estructurales de los cuatro marcos alineados en cada dirección del edificio. La resistencia nominal de los materiales son $f_{c}^{\prime}$ de $250 \mathrm{~kg} / \mathrm{cm}^{2}$ para el concreto y $f_{y}$ de $4200 \mathrm{~kg} / \mathrm{cm}^{2}$ para el acero de refuerzo.

Tabla 1. Movimientos del terreno considerados para el Estado Límite de Seguridad

\begin{tabular}{cccccc}
\hline Identificción & Zona de registro & Fecha & $\begin{array}{c}A M T^{l} \\
\left(\mathrm{~cm} / \mathrm{s}^{2}\right)\end{array}$ & $\begin{array}{c}V M T^{2} \\
\left(\mathrm{~cm} / \mathrm{s}^{2}\right)\end{array}$ & $\begin{array}{c}T_{g} \\
(\mathrm{seg})\end{array}$ \\
\hline mx01 & Alameda EO & $04 / 25 / 89$ & 45.83 & 15.103 & 2.1 \\
mx03 & Garibaldi EO & $04 / 25 / 89$ & 52.24 & 17.484 & 2.2 \\
mx04 & Tlahuac EO & $09 / 19 / 85$ & 117.63 & 34.575 & 2.1 \\
mx06 & Tlahuac NS & $09 / 21 / 85$ & 49.26 & 13.108 & 2.0 \\
mx07 & Tlahuac EO & $09 / 21 / 85$ & 51.47 & 15.098 & 1.9 \\
mx08 & SCT EO & $09 / 19 / 85$ & 167.26 & 61.074 & 2.0 \\
\hline
\end{tabular}

${ }^{T}$ Aceleración máxima del terreno original

${ }^{2}$ Velocidad máxima del terreno original 
Tabla 2. Movimientos del terreno considerados para el Estado Límite de Servicio

\begin{tabular}{cccccc}
\hline Identificación & $\begin{array}{c}\text { Zona del } \\
\text { registro }\end{array}$ & Fecha & $\begin{array}{c}A M T^{l} \\
\left(\mathrm{~cm} / \mathrm{seg}^{2}\right)\end{array}$ & $\begin{array}{c}V M T^{2} \\
(\mathrm{~cm} / \mathrm{seg})\end{array}$ & $\begin{array}{c}T_{g} \\
(\mathrm{seg})\end{array}$ \\
\hline S43 & Garibaldi EO & $10 / 12 / 94$ & 13.9 & 5.38 & 2.1 \\
S45 & Garibaldi EO & $14 / 09 / 95$ & 30.5 & 9.73 & 2.0 \\
S46 & Garibaldi NS & $14 / 09 / 95$ & 26.0 & 8.04 & 2.1 \\
S59 & Liverpool EO & $09 / 10 / 95$ & 16.5 & 6.19 & 2.1 \\
S77 & Tlatelolco EO & $10 / 12 / 94$ & 14.9 & 4.58 & 2.1 \\
S79 & Tlatelolco EO & $14 / 09 / 95$ & 26.7 & 7.91 & 2.0 \\
S89 & Alameda EO & $14 / 09 / 95$ & 40.6 & 10.54 & 2.0 \\
S119 & C.U Juárez EO & $10 / 12 / 94$ & 14.8 & 4.47 & 1.9 \\
S129 & Cibeles EO & $09 / 10 / 95$ & 14.3 & 5.11 & 2.0 \\
S131 & Angares EO & $14 / 09 / 95$ & 29.6 & 9.50 & 1.6 \\
S143 & Tlatelolco NS & $14 / 09 / 95$ & 19.1 & 8.38 & 1.9 \\
S140 & SCT EO & $25 / 04 / 89$ & 40.0 & 19.45 & 2.0 \\
\hline
\end{tabular}
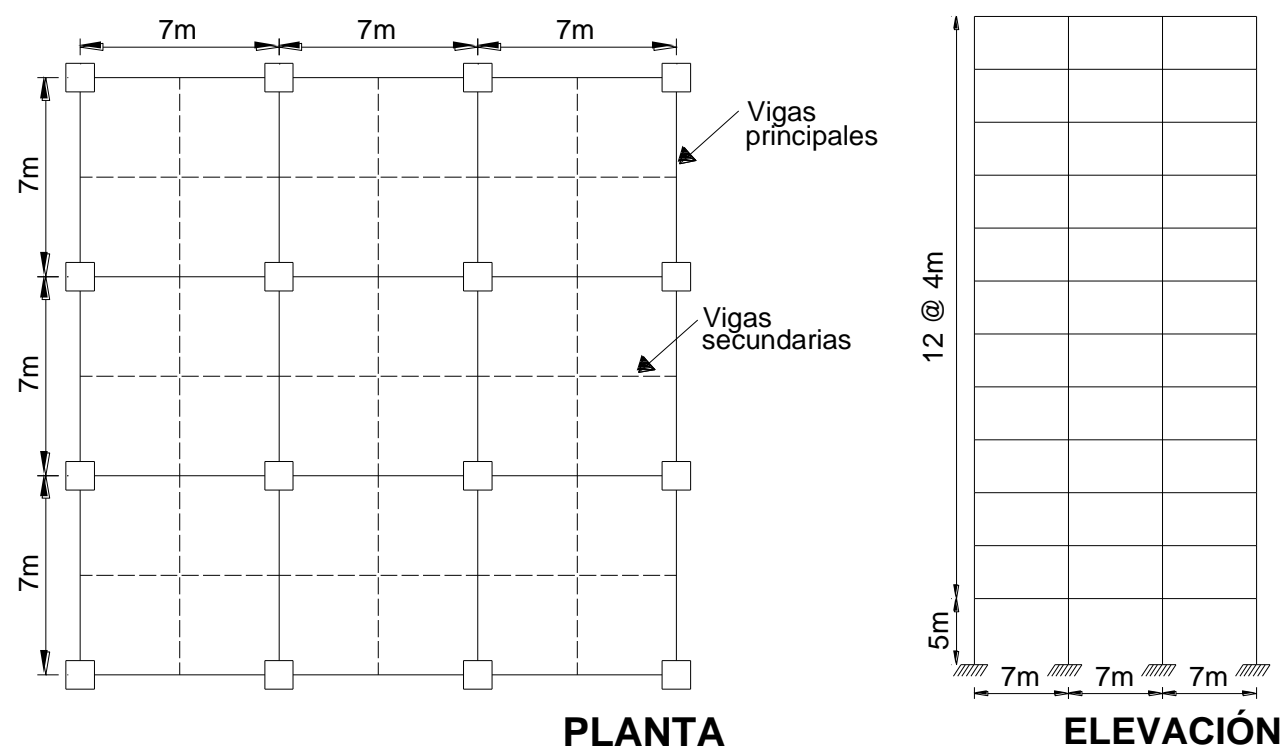

Figura 2. Planta y elevación del edificio

\section{Espectros de diseño}

La Figura 3 muestra los espectros de diseño correspondientes al estado límite de seguridad. En un primer paso se establecieron los espectros de resistencia y desplazamiento para diferentes valores de ductilidad acumulada constante, $\xi$ de 0.05 y comportamiento elasto-plástico perfecto. En un segundo paso, las ordenadas de los espectros, tanto de resistencia como de desplazamiento, fueron ajustados conforme a lo indicado por Terán y Espinoza (2008) para tomar en cuenta la degradación del ciclo histerético de las estructuras de concreto reforzado ubicadas en la Zona del Lago. La Figura 4 muestra los espectros de diseño para el estado límite de servicio, los cuales se establecieron para comportamiento elástico y $\xi$ de 0.02. Vale la pena mencionar que dentro de un contexto normativo, los espectros de diseño deben formularse de tal manera que tomen en cuenta la incertidumbre en la estimación de los periodos de la estructura y del movimiento del terreno. Esto por lo general resulta en que los espectros de diseño se 
definan de tal manera que exhiban una meseta relativamente ancha alrededor de la zona de máxima amplificación dinámica.
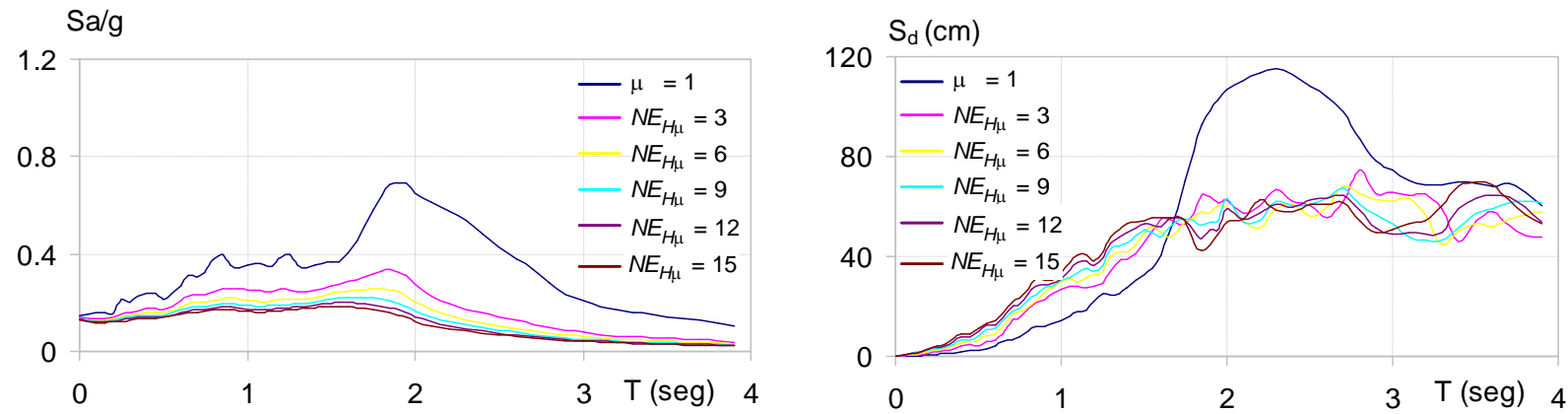

Figura 3. Espectros de resistencia y desplazamiento para el estado límite de seguridad
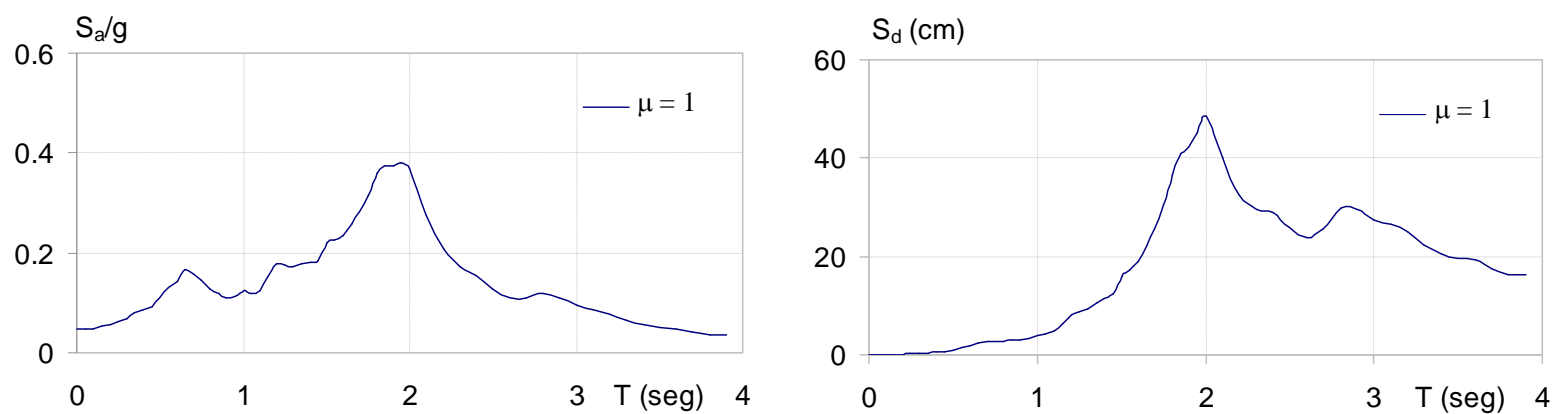

Figura 4. Espectros de resistencia y desplazamiento para el estado límite de servicio

Las ordenadas de pseudo-aceleración mostradas en las Figuras 3 y 4 se redujeron bajo la consideración que las estructuras sismorresistentes exhiben una sobrerresistencia con respecto a su resistencia lateral de diseño. En este estudio se consideran factores de sobrerresistencia de 1.5 y 1.2 para los estados límite de seguridad y servicio, respectivamente (mientras que las ordenadas originales del espectro de resistencia de seguridad se dividieron entre 1.5, las del estado límite de servicio se dividieron entre 1.2). En cuanto al valor de sobrerresistencia utilizado para el estado límite de seguridad, se consideró en primer lugar que varias investigaciones (Terán 1998) así como las recomendaciones del Apéndice A de las NTCDS sugieren que la resistencia lateral última en un marco dúctil de concreto reforzado se encuentra cercana al doble de su resistencia lateral de diseño. Para este estado límite, el factor de sobrerresistencia $(R)$ se define como la resistencia lateral última en la estructura normalizada para la resistencia lateral de diseño. Un valor de sobrerresistencia de dos implica que se espera que la resistencia lateral que la estructura desarrolla en su rango plástico de comportamiento se encuentre del orden del doble de la resistencia lateral para la cual se ha diseñado. En segundo lugar, Miranda (1991) propone la siguiente expresión para estimar el factor de sobrerresistencia:

$R=2-0.5 \sqrt{\frac{T}{5}}$

Para un edificio de doce pisos, como el considerado en este artículo, el periodo fundamental de vibración debe estar cercano a 1.2 segundos. La Ecuación 2 arroja para este valor de $T$ un factor de 
sobrerresistencia cercano a 1.75. Puede concluirse que el factor de sobrerresistencia para utilizarse para el estado límite de seguridad debe oscilar entre 1.75 y 2.0 cuando se utiliza el método estático de análisis estructural.

Recientemente, Terán (2004) observó que dentro del contexto de un diseño sísmico contra fatiga de bajo número de ciclos, es posible plantear el control de daño estructural de una edificación siempre y cuando el cortante basal de la misma esté cercano al arrojado por el método estático de análisis. Esto sugiere que el uso del método dinámico de análisis debe ser tal que el cortante basal dinámico no resulte por debajo del que arroja el método estático. Considerando que en la Zona del Lago del D.F. la respuesta dinámica de las edificaciones está dominada por el modo fundamental de vibración, y que bajo estas circunstancias el cortante basal dinámico está cercano a $80 \%$ del cortante basal estático, un análisis dinámico arrojará un cortante similar a un análisis estático sólo si el factor de sobrreresistencia asociado al primer tipo de análisis se aproxima al $80 \%$ del factor utilizado para el segundo tipo de análisis. En otras palabras, el factor de sobrerresistencia para considerarse durante un análisis dinámico debe plantearse como $0.8 R=0.8(1.75$ a 2.0$)=1.4$ a 1.6. Dado que el ejemplo con que se ilustran los conceptos en este artículo utiliza el método dinámico de análisis, se consideró el valor intermedio de 1.5 para reducir el espectro de resistencia asociado al estado límite de seguridad.

En cuanto al estado límite de servicio, el factor de sobrerresistencia se asocia a la resistencia lateral de fluencia, y no a la última que la estructura alcanza en su rango plástico de comportamiento. Esto implica que el valor de $R$ asociado a servicio, que debe ser mayor que uno debido al uso de factores de carga y reducción de resistencia durante el diseño, debe ser menor que el valor de $R$ asociado al estado límite de seguridad. Dado que la única información que se disponía para definir el valor de $R$ para el estado límite de servicio era que este valor debería ser mayor que uno pero menor que 1.5, se seleccionó un valor de 1.2.

\section{Prediseño global}

Bajo la consideración que la edificación está estructurada con base en marcos dúctiles de concreto reforzado, se establece un valor de 5 para la ductilidad última. Se consideran además valores de $C O D$ de 1.2 y 1.6 para establecer los desplazamientos permisibles de azotea para los estados límite de servicio y seguridad, respectivamente:

$$
\begin{aligned}
& \delta_{S E G}=\frac{I D I_{S E G} H}{C O D_{S E G}}=\frac{0.015 \times 49}{1.6}=0.459 \mathrm{~m}=46 \mathrm{~cm} \\
& \delta_{S E R}=\frac{I D I_{S E R} H}{C O D_{S E R}}=\frac{0.004 \times 49}{1.2}=0.163 \mathrm{~m}=16.3 \mathrm{~cm}
\end{aligned}
$$

Respecto a los valores de COD que se han usado, Qi y Moehle (1991) observan que dicho valor oscila entre 1.5 y 2.0 en estructuras que ingresan de manera importante a su rango plástico de comportamiento. Además, el valor de $C O D$ tiende a incrementarse en estructuras con irregularidad de rigidez y resistencia lateral en altura, y conforme se incrementa el número de pisos de la edificación y la demanda de comportamiento plástico en la misma. Dado que el edificio por diseñarse aquí es regular en cuanto a sus propiedades estructurales en altura, se decidió usar un valor cercano al límite inferior definido por Qi y Moehle, que para el caso del edificio de doce pisos resultó de 1.6. En cuanto al valor de COD utilizado para servicio, se sabe que por definición tiene que ser mayor que uno, y menor que el valor utilizado para el estado límite de seguridad. Dado que no se disponía de mayor información, se utilizó un $C O D$ de 1.2 para el estado límite de servicio. 
A partir de los desplazamientos máximos de azotea, es posible establecer para cada estado límite un valor para el periodo fundamental de vibración de la estructura. Esto requiere corregir el desplazamiento de azotea por el factor $\alpha$, que depende del número de pisos y de la demanda máxima de ductilidad global en la edificación. Terán (2004) presenta un extenso estudio de los valores de $\alpha$ que exhiben marcos dúctiles y regulares de concreto reforzado de más de cuatro pisos. Observa que en marcos que permanecen elásticos, el valor de $\alpha$ tiende a 1.4, mientras que $\alpha$ tiende a 1.2 en estructuras que exhiben demandas moderadas y altas de ductilidad. Con base en lo anterior, se consideraron valores de $\alpha$ de 1.2 y 1.4, respectivamente, para los estados límite de seguridad y servicio:

$$
\begin{aligned}
& \frac{\delta_{S E G}}{\alpha_{S E G}}=\frac{46}{1.2}=38.33 \mathrm{~cm} \\
& \frac{\delta_{S E R}}{\alpha_{S E R}}=\frac{16.3}{1.4}=11.66 \mathrm{~cm}
\end{aligned}
$$

La Figura 5 muestra la determinación del periodo máximo permisible para cada estado límite considerado. Mientras que para servicio se obtiene un periodo de 1.4 segundos, a seguridad de vida le corresponde un periodo de 1.25 segundos. La condición crítica para el diseño local de rigidez lateral corresponde a: $T_{\max }=\operatorname{Menor}\left(T_{S E R} y T_{S E G}\right)=1.25 \mathrm{seg}$. Con el valor del periodo de diseño $\left(T_{\max }=1.25\right.$ seg), es posible establecer cuál de los dos espectros de resistencia mostrados en la Figura 6 debe utilizarse durante el análisis dinámico de la estructura. Dado que la ordenada del espectro asociado al estado límite de seguridad es mayor que la correspondiente al estado límite de servicio (ambas ordenadas evaluadas en $T_{\max }$ ), el análisis espectral modal de la estructura debe llevarse a cabo con el espectro de resistencia asociado el estado límite de seguridad.

Tan solo como referencia, el peso total del edificio de doce pisos es de 6200 toneladas. Si se considera que el factor de sobrerresistencia utilizado para reducir las ordenadas del espectro de resistencia asociado al estado límite de seguridad es de 1.5, entonces y acorde al coeficiente sísmico de 0.18 leído de la Figura 6b, el cortante basal esperado en la estructura puede estimarse como: $V_{b}=0.18 \times 1.5 \times 6200=$ 1680 toneladas. Si se considera que los cuatro marcos en cada dirección principal del edificio tienen las mismas dimensiones y armado, entonces el cortante basal esperado en cada marco puede estimarse como $1680 / 4=420$ toneladas.

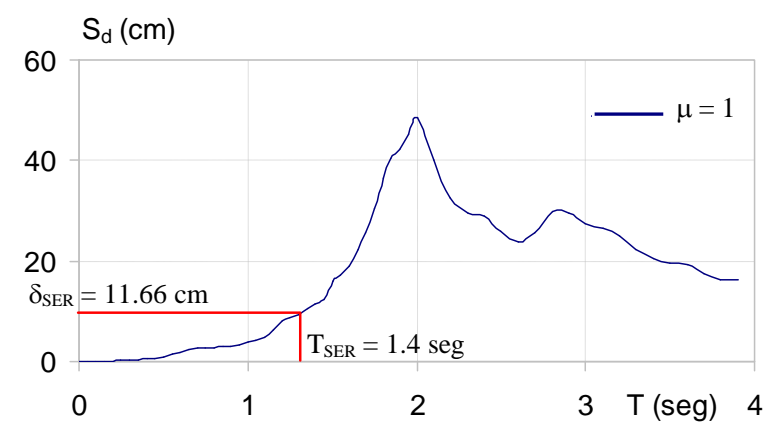

a) Servicio

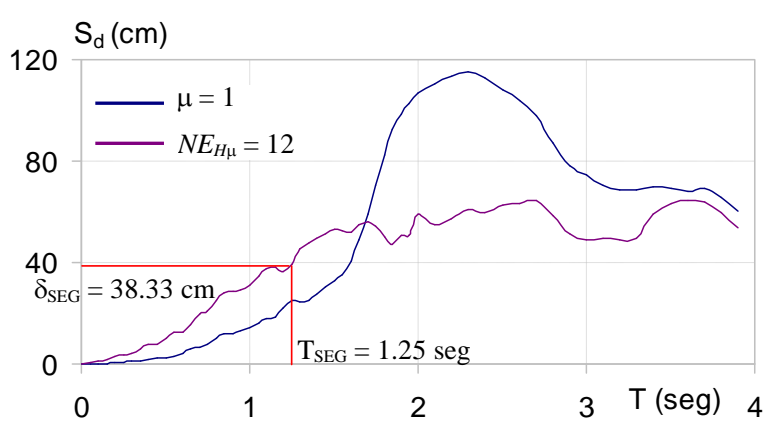

b) Seguridad

Figura 5. Determinación del periodo máximo permisible para servicio y seguridad 


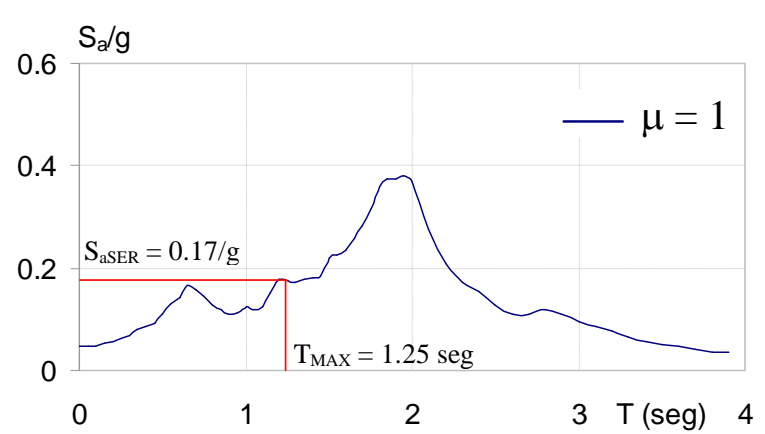

a) Servicio

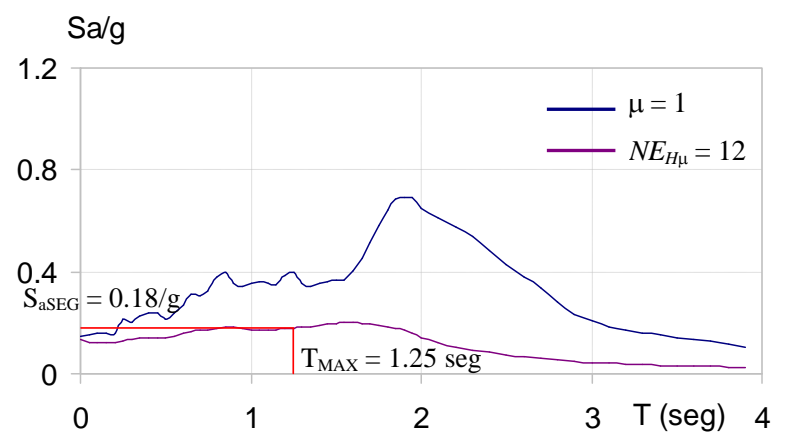

b) Seguridad

Figura 6. Determinación del cortante basal para servicio y seguridad

Una vez que se establecen los valores de los tres parámetros de diseño bajo consideración $\left(\mu_{u}=5\right.$, $T_{\max }=1.25 \operatorname{seg}$ y $V_{b}=1680$ toneladas) se procede al diseño local preliminar. Note que: A) El estado límite de seguridad rige el diseño de rigidez y resistencia del edificio; y B) El valor del cortante basal es solo una referencia, ya que se llevará a cabo un análisis dinámico del edificio con el espectro de resistencia correspondiente al estado límite de seguridad.

\section{Diseño Local Preliminar}

Conforme a la metodología propuesta, el primer paso del diseño local preliminar es el dimensionado de los elementos estructurales en función del valor de $T_{\max }$. Para el edificio bajo consideración, se realizó una serie de análisis de valores característicos con el programa SAP2000 para establecer las dimensiones de vigas y columnas. Dada la importancia de la contribución de la losa a la rigidez a flexión de las vigas, se consideró, a partir de las reglas establecidas por Pantazopoulou y French (2001), un ancho de losa tributario a las vigas. El agrietamiento de los elementos estructurales de concreto reforzado se consideró de acuerdo con las recomendaciones del FEMA 273 (Federal Emergency Management Agency 1997). Conforme a ellas, el momento de inercia de las vigas se tomó como el 50\% de su inercia gruesa, y el de las columnas como $70 \%$ de su inercia gruesa. Después de varias iteraciones, se establecieron las dimensiones resumidas en la Tabla 3. El proceso de dimensionado consideró el uso de secciones tipo para simplificar el procedimiento constructivo del edificio. Esto es particularmente cierto para las columnas, cuyo dimensionado se resolvió con una sola sección para todas ellas.

Tabla 3. Dimensiones de los elementos estructurales

\begin{tabular}{ccc}
\hline Niveles & Viga $(\mathrm{cm})$ & Columna $(\mathrm{cm})$ \\
\hline 1,2 y 3 & $50 \times 120$ & $90 \times 90$ \\
4,5 y 6 & $40 \times 120$ & $90 \times 90$ \\
7,8 y 9 & $40 \times 110$ & $90 \times 90$ \\
10,11 y 12 & $40 \times 100$ & $90 \times 90$ \\
\hline
\end{tabular}

A partir de las dimensiones mostradas en la Tabla 3 y la masa reactiva del edificio de doce pisos, se estimó un periodo fundamental de vibración de $1.24 \mathrm{seg}$, que es ligeramente menor que el valor de diseño $\left(T_{\max }=1.25 \mathrm{seg}\right)$.

El segundo paso del diseño preliminar local implica establecer las capacidades locales de resistencia. El análisis estructural del edificio se llevó a cabo mediante un análisis modal espectral con el 
programa SAP2000. Para ello se utilizó el espectro de resistencia de diseño correspondiente al estado límite de seguridad. Se consideraron dos condiciones de carga: $1.4(C M+C V)$ y $1.1(C M+C V \pm$ Sismo $)$. El diseño de las vigas del edificio se planteó de acuerdo a los requerimientos de las NTCDCEC. La Tabla 4 resume el acero longitudinal negativo y positivo en los extremos de las vigas, y sus correspondientes capacidades a flexión. Cabe mencionar que los valores de resistencia resumidos en la tabla consideran la contribución del acero de la losa. El suministro de acero longitudinal en columnas se realizó a partir de diagramas de interacción establecidos de acuerdo con las NTCDCEC. La Tabla 5 resume el acero longitudinal de las columnas. El hecho de que varias columnas posean el mismo armado se debe a los requisitos de acero mínimo.

Tabla 4. Propiedades Estructurales de vigas

\begin{tabular}{|c|c|c|c|c|c|c|c|}
\hline \multirow[b]{2}{*}{ Nivel de Piso } & \multicolumn{2}{|c|}{ Apoyo "a" } & \multicolumn{2}{|c|}{ Aроуо "b" } & & & \\
\hline & $\mathrm{M}^{-}$ & $\mathrm{M}^{+}$ & $\mathrm{M}^{-}$ & $\mathrm{M}^{+}$ & & & \\
\hline \multirow{3}{*}{1,2 y 3} & & & & & & & \\
\hline & 7 \# 10 & $5 \# 10$ & $6 \# 10$ & 4 \# 10 & & & \\
\hline & 235.3 & 157.42 & 209.6 & 128.26 & & & \\
\hline \multirow{2}{*}{4,5 у 6} & $6 \# 10$ & $5 \# 8$ & 5 \# 10 & $6 \# 8$ & & & \\
\hline & 202.47 & 102.6 & 178 & 121.31 & & & \\
\hline \multirow[b]{2}{*}{7,8 y 9} & $5 \# 10$ & $5 \# 8$ & 5 \# 10 & $5 \# 8$ & & & \\
\hline & 162 & 03 & 162 & 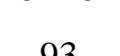 & & & \multirow{2}{*}{ b la } \\
\hline \multirow{3}{*}{10,11 y 12} & 102 & 93 & 102 & 93 & & & \\
\hline & $5 \# 8$ & $3 \# 8$ & $5 \# 8$ & $3 \# 8$ & \multirow{2}{*}{\multicolumn{3}{|c|}{$\begin{array}{c}\text { Nomenclatura de } \\
\text { apoyos }\end{array}$}} \\
\hline & 104.18 & 52 & 104.18 & 52 & & & \\
\hline
\end{tabular}

Tabla 5. Acero longitudinal en columnas

\begin{tabular}{ccc}
\hline Nivel de Piso & Columna Extremo & Columna Central \\
\hline 1,2 y 3 & $32 \# 8$ 36 \# 10 \\
4,5 y 6 & $16 \# 8$ & $20 \# 10$ \\
7,8 y 9 & $16 \# 8$ & $20 \# 8$ \\
10,11 y 12 & $16 \# 8$ & $20 \# 8$ \\
\hline
\end{tabular}

El tercer paso de acuerdo al diseño preliminar local es el detallado de los elementos estructurales. Este paso se llevó acorde a los requerimientos de detallado de las NTCDCEC para marcos dúctiles. Además de proveer resistencia a corte a los elementos estructurales, el detallado de los estribos es relevante para permitir que los elementos estructurales de concreto reforzado se comporten de manera estable en su rango plástico de comportamiento. Por cuestiones de espacio no se muestran los croquis de detallado.

\section{CARACTERÍSTICAS MECÁNICAS DEL EDIFICIO DE DOCE PISOS}

Una vez concluido el diseño preliminar, se procedió a estimar las características mecánicas del edificio de doce pisos a partir de un modelo de análisis no lineal. En resumen, se estableció un modelo 
bidimensional del marco central del edificio. Se contempló explícitamente el agrietamiento en las vigas y columnas, así como el efecto que la losa tiene en la resistencia, rigidez y capacidad de deformación de las vigas. Se utilizaron modelos analíticos para establecer las curvas de esfuerzo-deformación para el concreto confinado y no confinado, así como para el acero. A partir de estas curvas y de suponer que una sección plana permanece plana después de la flexión, se establecieron curvas momento-curvatura en ambos extremos de los elementos estructurales. Luego se establecieron idealizaciones bilineales de estas curvas por medio de definir curvaturas de fluencia y última. Una discusión detallada de las consideraciones utilizadas para modelar los elementos estructurales puede encontrarse en Terán y Bertero (1993).

El momento de fluencia de una sección, $M_{y}$, y su correspondiente curvatura, $\varphi_{y}$, se definieron como el momento y la curvatura en que alguna de las barras longitudinales de la sección exhibe fluencia incipiente. La curvatura última, $\varphi_{u}$, se definió como la menor curvatura que corresponde a las dos condiciones siguientes: A) El concreto de la sección alcanza su deformación unitaria última a compresión (aplastamiento); o B) Una de las barras longitudinales a tensión alcanza su elongación última (fractura). El momento último de la sección, $M_{u}$, se definió como el momento flexionante que exhibe dicha sección cuando alcanza la curvatura última. Por medio de conectar a través de una línea recta el origen con el punto definido por $\left(M_{y}, \varphi_{y}\right)$, y de conectar este último con el punto definido por $\left(M_{u}, \varphi_{u}\right)$ a través de otra línea recta, se definió la idealización bilineal del diagrama momento-curvatura. Note que el pandeo del acero a compresión de la sección no fue considerado como criterio de falla, y que la condición última no representa la "rotura" física de la sección, sino un estado límite de utilidad.

La resistencia, rigidez y capacidad de deformación de los elementos estructurales se establecieron de manera directa de estas curvas bilineales. En particular, la resistencia de cada sección se caracteriza por su momento de fluencia $\left(M_{y}\right)$. En el caso de las vigas, se definieron dos capacidades a flexión, una para flexión positiva (sección T o L) y otra para flexión negativa (sección rectangular). En el caso de las columnas, se consideró explícitamente la interacción fuerza axial-momento flexionante. Mientras que la rigidez agrietada a flexión de las secciones se estimó directamente de la pendiente correspondiente a la porción elástica de la curva bilineal, su endurecimiento por deformación se estableció de la pendiente correspondiente a la región plástica de la curva. La rigidez a flexión en cada extremo de las vigas se consideró igual al promedio de las rigideces positiva y negativa en ese extremo. La rigidez a flexión de una viga completa se estimó como el promedio de las rigideces correspondientes a sus dos extremos. Finalmente, la capacidad rotacional última en los extremos de los elementos estructurales fue caracterizada por:

$$
\theta_{u}=\left(\varphi_{u}-\varphi_{y}\right) L_{p}
$$

donde $\theta_{u}$ es la capacidad última de rotación de la sección, y $L_{p}$ la longitud de articulación plástica (que se supuso igual a la mitad del peralte efectivo de la sección).

La porción de la losa que interactúa, tanto en tensión como compresión, con las vigas se definió a partir de las recomendaciones de Pantazopoulou y French (2001) para una distorsión de 2\%. Cabe mencionar que las propiedades de los materiales estructurales utilizadas para estimar las propiedades estructurales de vigas y columnas no son las de diseño, sino las esperadas; y que para el caso particular del acero del refuerzo longitudinal se utilizaron las propiedades reportadas por Rodríguez y Botero (1997). Las columnas del primer piso se modelaron como empotradas en su base, y se consideró tanto el endurecimiento por deformación en las vigas como los efectos de segundo orden derivados de la carga gravitacional. 
La Tabla 6 resume las propiedades estructurales de las vigas de los marcos del edificio. Las unidades de rotación son radianes y las de curvatura $1 / \mathrm{m}$. Para obtener la inercia del elemento estructural $\left(I_{e}\right)$ se promediaron las inercias agrietadas positiva y negativa estimadas en los extremos de los elementos.

Una herramienta útil para evaluar las características mecánicas globales de una estructura es un análisis estático no lineal bajo deformación lateral monótonamente creciente (pushover). Este tipo de análisis consiste en aplicar una serie de cargas laterales con valor relativo constante en altura, hasta alcanzar la capacidad última de deformación del edificio. En este estudio se utilizó un patrón de carga proporcional a las fuerzas laterales de diseño derivadas del análisis dinámico modal. El análisis pushover se llevo a cabo con el programa DRAIN 2DX (Prakash et al. 1993). La Figura 7a muestra la curva desplazamiento de azotea contra cortante basal del marco central del edificio de doce niveles.

Tabla 6. Propiedades estructurales de las vigas

\begin{tabular}{|c|c|c|c|c|}
\hline \multirow[b]{2}{*}{ Nivel de Piso } & \multicolumn{2}{|c|}{ Aроуо "a" } & \multicolumn{2}{|c|}{ Apoyo "b" } \\
\hline & $\begin{array}{c}M \\
(\text { Ton-m) }\end{array}$ & $\begin{array}{c}M^{+} \\
(\text {Ton-m) }\end{array}$ & $\begin{array}{c}M \\
(\text { Ton-m) }\end{array}$ & $\begin{array}{c}M^{+} \\
(\text {Ton- } m)\end{array}$ \\
\hline \multirow{6}{*}{1,2 y 3} & $M_{y}=319.40$ & $M_{y}=192.90$ & $M_{y}=283.70$ & $M_{y}=154.80$ \\
\hline & $\theta=0.028$ & $\theta^{+}=0.066$ & $\theta=0.026$ & $\theta^{+}=0.066$ \\
\hline & End $^{-}=0.021$ & End $^{+}=0.014$ & End $^{-}=0.020$ & $\operatorname{End}^{+}=0.013$ \\
\hline & $\phi_{y}=0.00312$ & $\phi_{y}^{+}=0.00243$ & $\phi_{y}=0.00307$ & $\phi_{y}^{+}=0.00238$ \\
\hline & $\phi_{u}=0.05061$ & $\phi_{u}^{+}=0.11302$ & $\phi_{u}=0.04625$ & $\phi_{u}^{+}=0.11275$ \\
\hline & \multicolumn{2}{|c|}{$I_{e} / I_{g}=0.488$} & \multicolumn{2}{|c|}{$I_{e} / I_{g}=0.426$} \\
\hline \multirow{6}{*}{4,5 y 6} & $M_{y}=274.60$ & $M_{y}=124.80$ & $M_{y}=241.90$ & $M_{y}=149.50$ \\
\hline & $\theta=0.021$ & $\theta^{+}=0.066$ & $\theta=0.026$ & $\theta^{+}=0.066$ \\
\hline & End $^{-}=0.016$ & $E n d^{+}=0.013$ & End $^{-}=0.020$ & End $^{+}=0.013$ \\
\hline & $\phi_{y}=0.00323$ & $\phi_{y}^{+}=0.00233$ & $\phi_{y}=0.00308$ & $\phi_{y}^{+}=0.00237$ \\
\hline & $\phi_{u}=0.03788$ & $\phi_{u}^{+}=0.11187$ & $\phi_{u}=0.04674$ & $\phi_{u}^{+}=0.11246$ \\
\hline & \multicolumn{2}{|c|}{$I_{e} / I_{g}=0.444$} & \multicolumn{2}{|c|}{$I_{e} / I_{g}=0.452$} \\
\hline \multirow{6}{*}{7,8 y 9} & $M_{y}=218.30$ & $M_{y}=113.20$ & $M_{y}=218.30$ & $M_{y}=113.20$ \\
\hline & $\theta=0.023$ & $\theta^{+}=0.067$ & $\theta=0.023$ & $\theta^{+}=0.067$ \\
\hline & End $^{-}=0.018$ & $\operatorname{End}^{+}=0.013$ & End $^{-}=0.018$ & $\operatorname{End}^{+}=0.013$ \\
\hline & $\phi_{y}=0.00348$ & $\phi_{y}^{+}=0.00258$ & $\phi_{y}=0.00348$ & $\phi_{y}^{+}=0.00258$ \\
\hline & $\phi_{u}=0.04495$ & $\phi_{u}^{+}=0.12394$ & $\phi_{u}=0.04495$ & $\phi^{+}{ }_{u}=0.12394$ \\
\hline & \multicolumn{2}{|c|}{$I_{e} / I_{g}=0.436$} & \multicolumn{2}{|c|}{$I_{e} / I_{g}=0.436$} \\
\hline \multirow{6}{*}{10,11 y 12} & $M y=137.9$ & $M y=61.70$ & $M y=137.9$ & $M y=61.70$ \\
\hline & $\theta-=0.020$ & $\theta+=0.067$ & $\theta-=0.020$ & $\theta+=0.067$ \\
\hline & End $-=0.018$ & $E n d+=0.012$ & End $-=0.018$ & End $+=0.012$ \\
\hline & $\phi_{y}=0.00368$ & $\phi_{y}^{+}=0.00278$ & $\phi_{y}=0.00368$ & $\phi_{y}^{+}=0.00278$ \\
\hline & $\phi_{u}=0.04431$ & $\phi_{u}^{+}=0.13716$ & $\phi_{u}=0.04431$ & $\phi_{u}^{+}=0.13716$ \\
\hline & \multicolumn{2}{|c|}{$\mathrm{Ie} / \mathrm{Ig}=0.333$} & \multicolumn{2}{|c|}{$\mathrm{Ie} / \mathrm{Ig}=0.333$} \\
\hline
\end{tabular}

$M_{y}$ momento de fluencia

$\theta^{+} y \theta^{-}$rotaciones últimas positiva y negativa, respectivamente

End $^{+}$y End endurecimiento por deformación positivo y negativo, respectivamente

$\phi_{y}{ }^{+} \phi_{y}{ }^{-}$curvaturas de fluencia positiva y negativa, respectivamente

$\phi_{u}{ }^{+} \phi_{u}{ }^{-}$curvaturas ultimas positiva y negativa, respectivamente

$I_{e} / I_{g}$ momento de inercia agrietado normalizado por momento de inercia de la sección bruta 
De acuerdo con el análisis estático no lineal llevado a cabo con el programa DRAIN 2DX, el marco es capaz de desarrollar un cortante último de 390 toneladas. El cortante basal objetivo o esperado es de 420 toneladas por marco, de tal manera que el procedimiento de prediseño local seguido en este artículo resulta en un ligero sub-diseño de la resistencia lateral. En particular, se consideró durante el diseño un factor de sobrerresistencia de 1.5 respecto al coeficiente sísmico evaluado para $T_{\max }$ (Figura $7 \mathrm{~b}$ ), mientras que el factor de sobrerresistencia estimado a partir del pushover es:

$$
R=\frac{V_{\text {bREAL }}}{V_{\text {bESTATICO }}}=\frac{390 \text { ton }}{0.18(1550) \text { ton }}=1.40
$$

Si se considera que el cortante de diseño dinámico es alrededor de 0.80 veces el producto del coeficiente sísmico (leído directamente del espectro de resistencia) y del peso reactivo de la estructura, entonces el factor de sobrerresistencia respecto al cortante dinámico es:

$$
R=\frac{V_{\text {bREAL }}}{V_{\text {bDINAMICO }}} \approx \frac{390 \text { ton }}{0.8 \times 0.18(1550) \text { ton }}=1.75
$$

La capacidad de deformación última global del marco se determinó con base en una idealización bilineal de la curva cortante basal contra desplazamiento de azotea. Conforme a lo mostrado en la Figura 7a, el desplazamiento de fluencia global se define como el desplazamiento que delimita la zona elástica y plástica de la curva bilineal y, en el caso del edificio de doce pisos, es igual a $17 \mathrm{~cm}$. El desplazamiento último global se define como aquel en el que se agota la capacidad de deformación rotacional de por lo menos una tercera parte de las vigas de cualquier entrepiso. Para el edificio de doce pisos, el desplazamiento último de azotea es igual a $80 \mathrm{~cm}$, lo que implica una ductilidad última global de $\mu_{u}=\delta_{u} /$ $\delta_{y}=0.80 / 0.17=4.7$. Este valor es similar al valor de 5 considerado durante el prediseño global del marco, lo que implica que el detallado especificado por las NTCDCEC para marcos dúctiles ha resultado en un diseño que exhibe una ductilidad global dentro del rango esperado (4 a 6). En la Figura $7 \mathrm{~b}$ es posible observar la evolución del $C O D$ del marco de doce pisos en función de un incremento en su desplazamiento de azotea $\left(\delta_{a z t}\right)$. Puede concluirse que los valores de $C O D$ utilizados durante la etapa de prediseño global son congruentes con los mostrados en la Figura $7 \mathrm{~b}$.

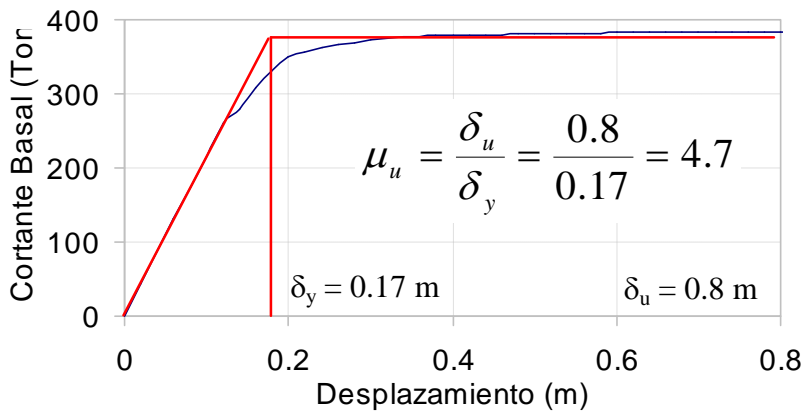

a) Pushover DRAIN 2DX

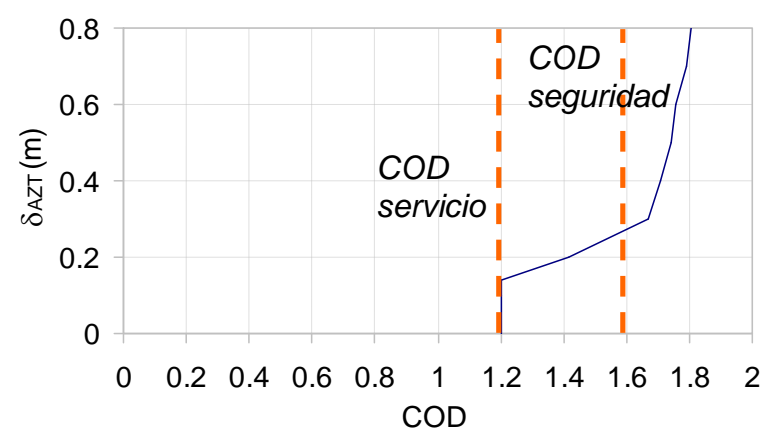

b) COD vs. $\delta_{\mathrm{AZT}}$

Figura 7. Comportamiento del edificio de 12 pisos en función de su desplazamiento de azotea

Aunque este hecho no se ilustra, el mecanismo plástico del edificio corresponde al de viga débilcolumna fuerte. Puede decirse que los requerimientos de diseño y detallado de las NTCDCEC para marcos dúctiles han dado lugar a una estructura con comportamiento plástico estable. 
La Figura 8 muestra, de acuerdo a los resultados obtenidos del análisis estático no lineal del edificio, las distribuciones en altura de desplazamiento lateral y distorsión de entrepiso para desplazamientos de azotea de 20, 40,60 y $80 \mathrm{~cm}$. Estas distribuciones abarcan el comportamiento de la estructura desde su fluencia incipiente hasta su capacidad última de deformación lateral. Las distorsiones de entrepiso correspondiente a fluencia incipiente $(20 \mathrm{~cm}$ de desplazamiento de azotea) muestran una distribución razonablemente uniforme en altura con valores máximos ligeramente mayores que 0.005. Note que los valores de distorsión en que según el modelo analítico ocurre la fluencia incipiente de los elementos estructurales son congruentes con el valor de 0.005 ofrecido por Reyes (2000).

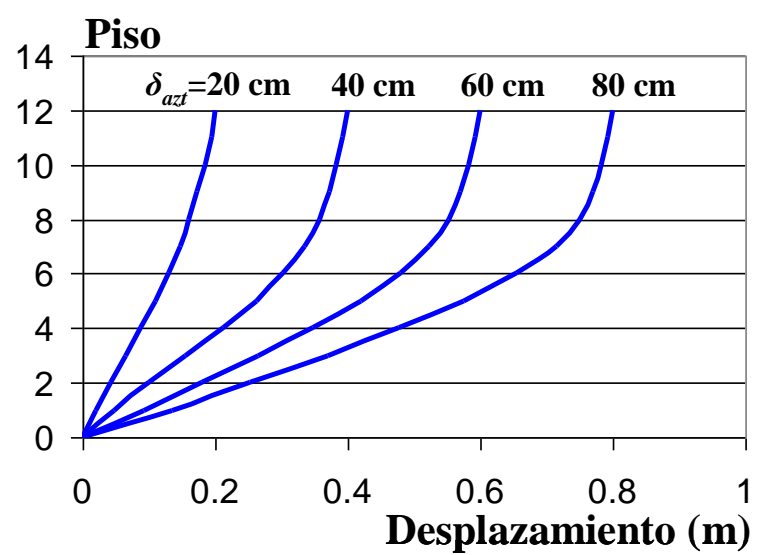

a) Desplazamiento de piso

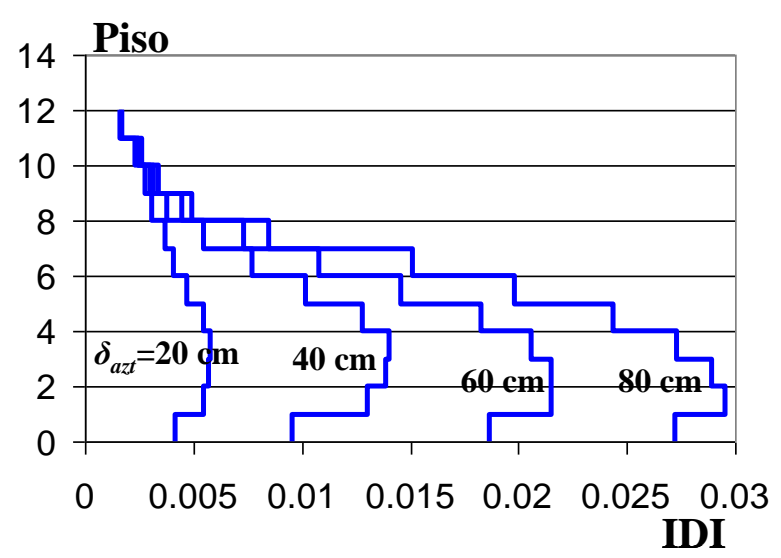

b) Distorsión de entrepiso

Figura 8. Evolución de la distribución de deformación lateral en altura

La distribución de deformaciones en altura cambia conforme la demanda global de comportamiento plástico se incrementa. En particular, conforme el desplazamiento de azotea se incrementa hasta alcanzar un valor de $80 \mathrm{~cm}$, los niveles inferiores empiezan a acumular mayor deformación lateral respecto a los niveles superiores. Para un desplazamiento de azotea de $80 \mathrm{~cm}$, se observa una notoria acumulación de distorsión en la mitad inferior del edificio, y una distorsión máxima cercana a 0.03. El valor de distorsión en que el modelo analítico predice la falla incipiente de los elementos estructurales es congruente con el valor de 0.04 ofrecido por Reyes (2000).

Las Figuras 7 y 8 reflejan el impacto de los objetivos de diseño en las características mecánicas del edificio. En cuanto al estado límite de servicio, los objetivos de diseño indican que mientras que los elementos estructurales deben permanecer en lo esencial elásticos, el desempeño no estructural adecuado se promueve a partir de controlar la distorsión máxima de entrepiso dentro del umbral de 0.004 (ver Figura 1). De acuerdo con la Figura 7a, el desplazamiento de fluencia idealizado del edificio es de $17 \mathrm{~cm}$, de tal manera que controlar el desplazamiento de azotea dentro del umbral de $16.3 \mathrm{~cm}$ obtenido mediante la Ecuación 3b promueve que los elementos estructurales permanezcan elásticos. Note en la Figura 8b que la distorsión máxima para un desplazamiento de azotea de $20 \mathrm{~cm}$ es del orden de 0.0055 . Considerando que sea razonable estimar la distorsión máxima para un desplazamiento de azotea de $16.3 \mathrm{~cm}$ por medio de considerarla proporcional a la correspondiente a $20 \mathrm{~cm}$, se tiene que $I D I_{S E R}=(0.0055) 16.3 / 20=0.0045$, valor que es congruente con el umbral de 0.004 .

En cuanto al estado límite de seguridad, los objetivos de diseño indican que las demandas acumuladas de deformación plástica deben ser controladas para evitar colapsos locales. Conforme 
observan Teran y Espinoza (2008), esto implica para una estructura sujeta a demandas severas de energía que la ductilidad máxima se limite a valores cercanos al 50\% del valor de su ductilidad última. En el caso del edificio de doce pisos, el 50\% de la capacidad última de deformación es igual a $40 \mathrm{~cm}$, valor que es similar al umbral de $46 \mathrm{~cm}$ establecido mediante la Ecuación 3a. Conforme a la Figura 1, el desempeño no estructural adecuado se promueve a partir de controlar la distorsión máxima de entrepiso dentro del umbral de 0.015. De acuerdo con la Figura 8b, la distorsión máxima para un desplazamiento de azotea de $40 \mathrm{~cm}$ es del orden de 0.014. Considerando que sea razonable estimar la distorsión máxima para un desplazamiento de azotea de $46 \mathrm{~cm}$ por medio de considerarla proporcional a la correspondiente a un desplazamiento de azotea de $40 \mathrm{~cm}$, se tiene que $I D I_{S E G}=(0.014)^{46} / 40=0.0160$, valor que es congruente con el umbral de 0.0150. Puede concluirse que para el estado límite de seguridad, el desempeño no estructural y las demandas acumuladas de deformación plástica resultan en que el edificio deba controlar su desplazamiento de azotea dentro del umbral de $46 \mathrm{~cm}$, y que por tanto, no pueda desarrollar durante la excitación sísmica de diseño su capacidad última de deformación (que corresponde a un desplazamiento de azotea de $80 \mathrm{~cm}$ ). En cuanto al periodo fundamental de vibración del edificio, el programa DRAIN 2DX arroja un valor de $1.30 \mathrm{seg}$.

De acuerdo con lo presentado hasta el momento, puede decirse que la metodología de prediseño local utilizada resulta en que las propiedades estructurales globales del edificio de doce pisos sean razonablemente similares a los valores implicados por los parámetros de diseño obtenidos durante la etapa de prediseño global (ver Tabla 7).

Tabla 7. Características mecánicas globales del marco de doce pisos

\begin{tabular}{ccc}
\hline Característica Mecánica & Parámetro de Diseño & Valor derivado de análisis pushover \\
\hline Rigidez & $T_{\max }=1.25 \mathrm{seg}$ & $T_{\max }=1.3 \mathrm{seg}$ \\
$\begin{array}{c}\text { Resistencia } \\
\begin{array}{c}\text { Capacidad de } \\
\text { deformación }\end{array}\end{array}$ & $V_{b}=420$ ton/marco & $\boldsymbol{V}_{u}=490 \mathrm{ton} / \mathrm{marco}$ \\
Mecanismo & $\begin{array}{c}\text { Viga débil-columna } \\
\text { fuerte }\end{array}$ & Viga débil-columna fuerte \\
\hline
\end{tabular}

\section{DESEMPEÑO SISMICO DEL EDIFICIO DE DOCE PISOS}

Una vez establecidas las características mecánicas globales del marco, es necesario revisar su desempeño sísmico conforme a sus objetivos de diseño. En este capítulo se revisa el desempeño sísmico del edificio de doce pisos para los estados límite de servicio y seguridad. Dado que para los dos estados límite bajo consideración se espera agrietamiento extensivo de los elementos estructurales, se utiliza el mismo modelo de análisis no lineal planteado con anterioridad. Note que si el nivel de agrietamiento esperado para los estados límite fuera diferente, sería necesario establecer dos modelos de análisis no lineal que reflejen las condiciones particulares asociadas al nivel de daño esperado en cada estado límite. Para el caso de los análisis dinámicos no lineales, se adaptó el modelo planteado para el análisis estático no lineal de tal manera que considerara el amortiguamiento viscoso a través de una matriz de amortiguamiento de Rayleigh, con 5\% de amortiguamiento crítico para los dos primeros modos.

Por un lado, quizá valga la pena mencionar que la aproximación más fuerte que resulta del uso del mismo modelo para ambos estados límite sea que para las distorsiones que se espera para el estado límite de servicio, el ancho de losa que contribuye a las propiedades estructurales de las vigas del marco será 
menor que la indicada por Pantazopoulou y French (2001). Para el estado límite de seguridad, las distorsiones esperadas son congruentes con las consideradas por Pantazopoulou y French para establecer el ancho de losa. Por el otro lado, las vigas del edificio de doce pisos son muy robustas y poseen un armado longitudinal importante, de tal manera que en lo general el ancho de la losa que se considere para establecer sus propiedades estructurales no afecta de manera importante el valor de dichas propiedades.

\section{Estado límite de servicio}

Mientras que para el estado límite de servicio se planteó el control del daño no estructural a partir de controlar la distorsión máxima de entrepiso dentro del umbral de 0.004, el control del daño estructural se planteó a través de mantener a los elementos estructurales dentro de su rango elástico de comportamiento. Para evaluar el desempeño del marco de doce pisos para el estado límite de servicio, se sometió al modelo del DRAIN 2DX a la familia de acelerogramas resumida en la Tabla 2. La Figura 9a muestra los valores de distorsión máxima para cada acelerograma, y compara la media más una desviación estándar de la distorsión máxima con su correspondiente valor de diseño. El número que se ha asignado a cada diamante de la figura corresponde al número de piso del edificio donde se presentó la distorsión máxima.

Mientras que la mayoría de los valores de distorsión máxima están por debajo de la distorsión de diseño, la media más una desviación estándar, que está cercana a 0.003 , no excede el umbral de diseño de 0.004. De acuerdo a esto, puede concluirse que el desempeño no estructural del marco satisface los requerimientos impuestos por los objetivos de diseño. En cuanto al desempeño estructural, los elementos estructurales permanecieron elásticos durante todos los acelerogramas considerados en el estudio.

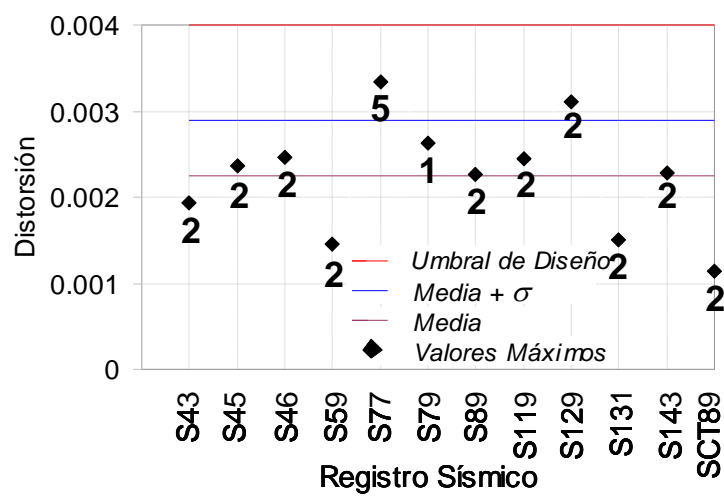

a) Estado Limite de Servicio

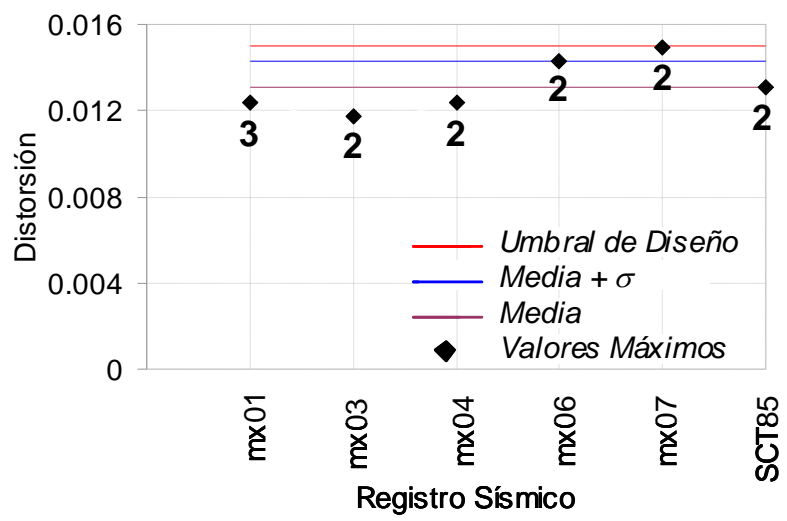

b) Estado Limite de Seguridad

Figura 9. Distorsiones máximas para los estados límite de servicio y seguridad

\section{Estado límite de seguridad}

Mientras que para el estado límite de seguridad de vida el control del daño no estructural se planteó a partir de controlar la distorsión máxima de entrepiso dentro del umbral de 0.015 , el control del daño estructural se planteó a partir de controlar las demandas locales de comportamiento plástico de tal manera que el valor de un índice de daño no exceda el valor de uno. Para evaluar el desempeño del marco de doce pisos, se sometió al modelo del DRAIN $2 D X$ a la familia de acelerogramas resumida en la Tabla 1 . Con este propósito se utilizó una versión modificada del programa DRAIN 2DX (Ascheim 2005) que 
contempla la degradación del ciclo histéretico de los elementos estructurales a través del modelo de Takeda (1970). La Figura 9b muestra los valores de distorsión máxima para cada acelerograma, y compara la media más una desviación estándar de la distorsión máxima con su correspondiente valor de diseño. Dado que todos los valores de distorsión máxima están por debajo de la distorsión de diseño, la media más una desviación estándar no excede el umbral de diseño. De acuerdo con esto, puede concluirse que el desempeño no estructural del marco satisface los requerimientos impuestos por los objetivos de diseño.

El daño a nivel local (a nivel elemento) se evalúa en los extremos de las vigas, donde se supone se concentran las mayores demandas de comportamiento plástico. La estimación del daño se realizó a través de una versión modificada del índice de Park y Ang (Terán 1998):

$$
D M I_{P A}=\max \left(\frac{\phi_{\max }^{+}}{\phi_{u}^{+}}, \frac{\phi_{\max }^{-}}{\phi_{u}^{-}}\right)+\beta\left(\frac{E_{H}^{+}}{M_{y}^{+} \phi_{u}^{+}}+\frac{E_{H}^{-}}{M_{y}^{-} \phi_{u}^{-}}\right)
$$

donde $\phi_{u}$ es la capacidad última de curvatura del elemento, $\phi_{\max }$ es la curvatura máxima demandada por la excitación sísmica, $E_{H}$ es la energía plástica disipada por la articulación plástica y $M_{y}$ es el momento de fluencia. Un signo positivo implica flexión positiva; un signo negativo, flexión negativa. Se utilizó $\beta$ de 0.15 , valor que caracteriza la estabilidad del ciclo histerético de elementos con buen detallado sísmico. Se plantearon dos enfoques para establecer el nivel de daño estructural en las vigas del marco a partir de la Ecuación 8. El primer enfoque se basa en estimar el valor $D M I_{P A}$ en los dos extremos del elemento, y asignarle a este el mayor de estos dos valores. El segundo enfoque consiste en asignarle al elemento el valor promedio de los valores de $D M I_{P A}$ en sus extremos. El valor de $D M I_{P A}$ para un entrepiso se establece a partir de promediar los valores de $D M I_{P A}$ estimados para todas las vigas del mismo. La Figura 10 muestra la distribución de daño estructural que exhiben los diferentes entrepisos para los dos enfoques (media más una desviación estándar). Note que el mayor nivel de daño estructural tiende a presentarse en los primeros niveles del edificio. La media del daño en el entrepiso más dañado es de 0.62 , mientras que la media más una desviación estándar exhibe un valor cercano a 0.8. Dado que el último valor está por debajo del umbral definido por el valor de uno, puede considerarse que el marco satisface adecuadamente las condiciones de desempeño estructural impuestas por los objetivos de diseño.

Los resultados obtenidos durante la evaluación del desempeño del marco indican que los elementos estructurales más dañados son las vigas extremo del segundo nivel. La Figura 11 muestra la historia de histéresis para el extremo más dañado de la viga más dañada del marco. Las curvas de histéresis reflejan claramente la degradación de rigidez conforme se incrementa la magnitud de los ciclos de comportamiento plástico. Se concluye, a través de comparar la capacidad de rotación de la viga (Tabla 6) y las demandas máximas de rotación observadas en la Figura 11, que el resultado final del diseño del marco ha sido limitar las demandas máximas de deformación plástica dentro de valores que corresponden aproximadamente a la mitad de las capacidades últimas de deformación de la viga.

\section{Observaciones Finales}

Antes de concluir este artículo, los autores quisieran enfatizar dos puntos que consideran relevantes:

1. El valor de varios de los parámetros considerados en el ejemplo de aplicación ha sido estimado a partir de complementar información existente con juicio ingenieril. En particular, no existen estudios orientados a cuantificar el valor de parámetros tales como el coeficiente de distorsión y sobrerresistencia lateral para el estado límite de servicio. Para hacer posible la aplicación práctica 
de una metodología como la aquí propuesta, es necesario calibrar el valor de dichos parámetros para varios sistemas y materiales estructurales, así como para diferentes estados límite.

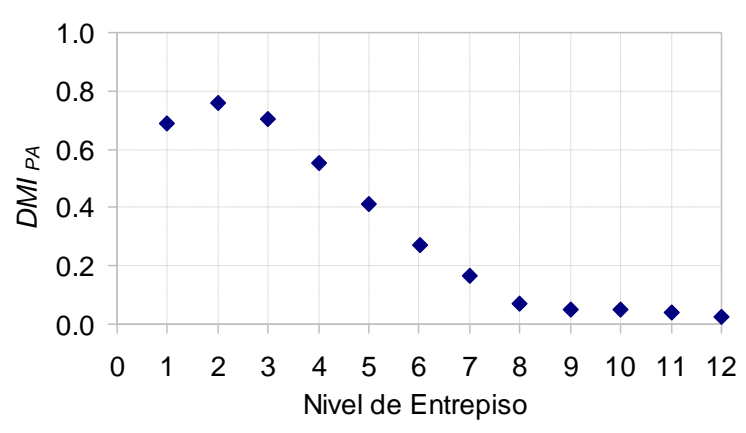

a) Enfoque 1

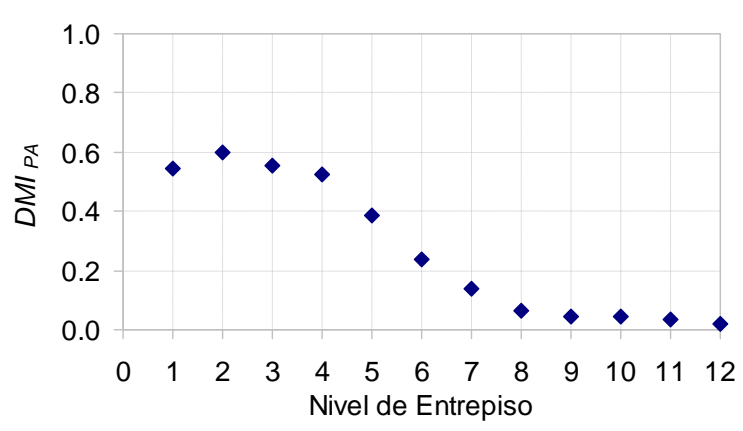

b) Enfoque 2

Figura 10. Distribución de daño en altura, estado límite de seguridad
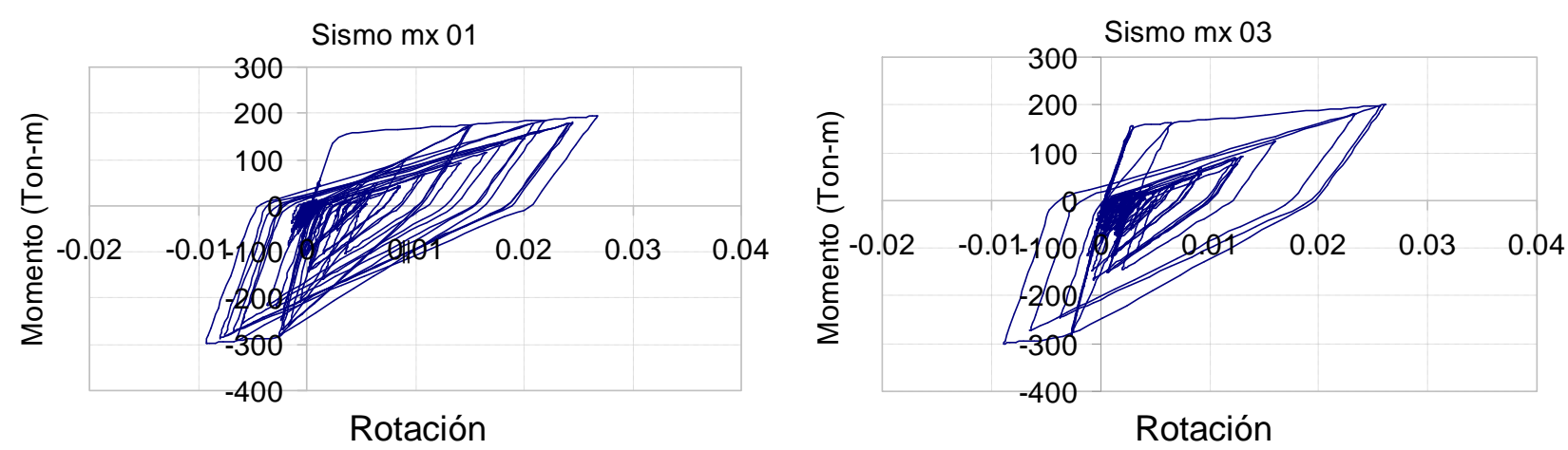

Figura 11. Historia de histéresis en el extremo mas dañado del marco, segundo nivel

2. Utilizar marcos momento-resistentes para edificaciones desplantadas en la Zona del Lago del D.F. y cuyo periodo fundamental de vibración se asemeje al periodo dominante del terreno, resulta en soluciones poco eficientes desde un punto de vista económico. Esto puede concluirse a partir de las dimensiones obtenidas para las vigas y columnas del edificio de doce pisos, así como de su armado. Para este tipo de estructuras, los sistemas estructurales duales (por ejemplo, los formados por marcos y muros de concreto reforzado) y los sistemas estructurales innovadores basados en la disipación pasiva de energía resultan en soluciones mas eficientes.

\section{CONCLUSIONES}

La metodología propuesta en este artículo ha sido aplicada exitosamente al diseño por desempeño de un edificio regular de doce pisos estructurado con base en marcos dúctiles de concreto reforzado. El desempeño sísmico esperado del edificio de doce pisos refleja adecuadamente el planteamiento hecho por sus objetivos de diseño. En particular, la distorsión de entrepiso máxima en el edificio se logró controlar, a través de la aplicación de la metodología de diseño propuesta, dentro de los umbrales establecidos de 
acuerdo al desempeño no estructural deseado. En cuanto al desempeño estructural, la metodología de diseño propuesta ha resultado en una estructura que es capaz de controlar sus demandas máxima y acumulada de deformación plástica de acuerdo al desempeño estructural deseado.

La metodología propuesta ha logrado incorporar exitosamente para el ejemplo desarrollado, las demandas acumuladas de deformación plástica a través del uso de espectros de ductilidad acumulada constante. La modificación que se ha planteado para dichos espectros para que contemplen explícitamente la degradación del ciclo histerético ha resultado en un diseño sísmico satisfactorio para el edificio de doce pisos. En cuanto al detallado de los elementos estructurales, puede decirse que el uso de los requerimientos de detallado de marcos dúctiles incluidos en las Normas Técnicas Complementarias para Diseño de Estructuras de Concreto del Reglamento de Construcciones del Distrito Federal ha dado lugar a que los marcos que constituyen el sistema estructural del edificio de doce pisos exhiban una capacidad de deformación última satisfactoria.

Una metodología como la introducida en este artículo puede ser la base para incorporar a la normatividad sísmica mexicana conceptos de diseño por desempeño. Los resultados obtenidos en este estudio abren expectativas para que los reglamentos actuales de diseño sísmico den lugar a diseños más confiables a través de modificar ligeramente su formato. La aplicación práctica de la metodología a otros materiales y sistemas estructurales requiere calibrar el valor de los diferentes parámetros involucrados en la misma para cada caso, y hacer un estudio más extenso de su aplicación a edificaciones y sistemas estructurales que reflejen la gran variedad de condiciones observadas en las estructuras reales.

\section{RECONOCIMIENTOS}

Los autores agradecen el apoyo de la Universidad Autónoma Metropolitana. El primer autor agradece al Consejo Nacional de Ciencia y Tecnología por la beca que le ha otorgado.

\section{REFERENCIAS}

Ascheim M (2005), http://nisee.berkeley.edu/software/drain2dx

Bertero R D y V V Bertero (1992), "Tall reinforced concrete buildings: conceptual earthquake-resistant design methodology", Reporte UCB/EERC-92/16, Universidad de California en Berkeley.

Chopra A. K. (2001), "Dynamics of Structures, Theory and applications to earthquake engineering", Editorial Prentice Hall, Segunda Edición.

Federal Emergency Management Agency (1997), "FEMA 273, NEHRP guidelines for the seismic rehabilitation of buildings".

Gobierno del Distrito Federal (2004a), "NORMAS TÉCNICAS COMPLEMENTARIAS PARA DISEÑO POR SISMO”, Gaceta Oficial del Distrito Federal, Décimo Cuarta Época, II (103-Bis), 55-77.

Gobierno del Distrito Federal (2004b), "NORMAS TÉCNICAS COMPLEMENTARIAS PARA DISEÑO Y CONSTRUCCIÓN DE ESTRUCTURAS DE CONCRETO", Gaceta Oficial del Distrito Federal, Décimo Cuarta Época, I (103-Bis), 88-194.

Miranda E (1991), "Seismic evaluation and upgrading of existing buildings", Tesis Doctoral, Universidad de California en Berkeley. 
Pantazopoulou S J y C W French (2001), "Slab participation in practical earthquake design of reinforced concrete frames", ACI Structural Journal, 98 (4), 479-489.

Park Y J y A H Ang (1985), "Mechanistic seismic damage model for reinforced concrete", ASCE Journal of Structural Engineering, 111(4), 722-739.

Prakash V, G H Powell y S Campbell (1993), "DRAIN-2DX Base program description and user guide”, Reporte UCB/SEMM-93/17, Universidad de California en Berkeley.

Priestley M J N (2000), "Performance based seismic design", Memorias $12^{\text {th }}$ World Conference on Earthquake Engineering, Nueva Zelanda, CDROM.

Qi X y J P Moehle (1991), "Displacement design approach for reinforced concrete structures subjected to earthquakes", Reporte No. UCB/EERC-91/02, Universidad de California en Berkeley.

Reyes Salinas C (2000), "El estado límite de servicio en el diseño sísmico de edificios”, Tesis Doctoral, División de Estudios de Posgrado de la Facultad de Ingeniería, UNAM.

Rodríguez M E y J C Botero (1997), "Evaluación del comportamiento de barras de acero de refuerzo sometidas a cargas monotónicas y cíclicas reversibles incluyendo pandeo", Revista de Ingeniería Sísmica, $56,9-27$.

Sánchez-Badillo A (2005), "Diseño por desempeño de marcos de concreto reforzado, de mediana y gran altura ubicados en la Zona del Lago del Distrito Federal", Tesis de Maestría en revisión. Posgrado en Ingeniería Estructural. División de Ciencias Básicas e Ingeniería, UAM-AZCAPOTZALCO.

SEAOC (1995), "Performance based seismic engineering of buildings", Vision 2000 Committee.

Takeda T, M A Sozen y N Nielsen (1970), "Reinforced Concrete Response to Simulated Earthquakes", ASCE Journal of Structural División, 96 (ST12).

Terán-Gilmore A y V V Bertero (1993), "Seismic performance of a 30-story building located on soft soil and designed according to UBC 1991", Reporte No. UCB/EERC-93/04, Universidad de California en Berkeley.

Terán-Gilmore A (1998), “Características mecánicas y desempeño sísmico de marcos dúctiles de concreto reforzado", Memorias del XI Congreso Nacional de Ingeniería Estructural, Monterrey.

Terán-Gilmore A y J O Jirsa (2003), "Un modelo simple para predecir la ocurrencia de fatiga de bajo número de ciclos", Memorias XIV Congreso Nacional de Ingeniería Sísmica, León, (CDROM).

Terán-Gilmore A y J O Jirsa (2004), "Uso de espectros de ductilidad acumulada constante para un diseño sísmico que contemple el efecto de la fatiga de bajo número de ciclos", Memorias XIV Congreso Nacional de Ingeniería Estructural, Acapulco, (CDROM).

Terán-Gilmore A (2004), "On the use of spectra to establish damage control in regular frames during global predesign", Earthquake Spectra, 20(3), 1-26.

Terán-Gilmore A y M Espinoza-Johnson (2008), "Diseño por desempeño de marcos dúctiles de concreto reforzado ubicados en la Zona del Lago del D.F.: la resistencia lateral de diseño", Revista de Ingeniería Sísmica, 78, 23-46. 